\title{
A Mobile Platform for Wireless Charging and Data Collection in Sensor Networks
}

\author{
Liguang Xie, Member, IEEE, Yi Shi, Senior Member, IEEE, Y. Thomas Hou, Fellow, IEEE, \\ Wenjing Lou, Fellow, IEEE, Hanif D. Sherali, Huaibei Zhou, and Scott F. Midkiff, Senior Member, IEEE
}

\begin{abstract}
Wireless energy transfer (WET) is a new technology that can be used to charge the batteries of sensor nodes without wires. Although wireless, WET does require a charging station to be brought to within reasonable range of a sensor node so that a good energy transfer efficiency can be achieved. On the other hand, it has been well recognized that data collection with a mobile base station has significant advantages over a static one. Given that a mobile platform is required for WET, a natural approach is to employ the same mobile platform to carry the base station for data collection. In this paper, we study the interesting problem of co-locating a wireless charger (for WET) and a mobile base station on the same mobile platform - the wireless charging vehicle (WCV). The WCV travels along a preplanned path inside the sensor network. Our goal is to minimize energy consumption of the entire system while ensuring that (i) each sensor node is charged in time so that it will never run out of energy, and (ii) all data collected from the sensor nodes are relayed to the mobile base station. We develop a mathematical model for this problem (OPT-t), which is timedependent. Instead of solving OPT-t directly, we show that it is sufficient to study a special subproblem (OPT-s) which only involves space-dependent variables. Subsequently, we develop a provably near-optimal solution to OPT-s. Our results offer a solution on how to use a single mobile platform to address both WET and data collection in sensor networks.
\end{abstract}

Index Terms-Wireless sensor network, wireless energy transfer, mobile base station, optimization.

\section{INTRODUCTION}

Recently, wireless energy transfer (WET) based on magnetic resonant coupling was shown to be a promising technology to fundamentally address energy and lifetime problems in a wireless sensor network (WSN) [9], [10], [21], [22]. Compared to other WET technologies such as electromagnetic radiation [5], [18], magnetic resonant coupling enjoys significant advantages. These advantages include: significantly higher energy transfer efficiency, immunity to the neighboring environment,

Manuscript received March 30, 2014; revised September 13, 2014; accepted December 16, 2014. An abridged version of this paper appeared in IEEE INFOCOM 2013 [25], Turin, Italy, April 14-19, 2013.

L. Xie, Y.T. Hou, W. Lou, H.D. Sherali, and S.F. Midkiff are with Virginia Tech, Blacksburg, VA, USA 24061 (e-mail: xie@vt.edu; thou@vt.edu; wjlou@vt.edu; hanifs@vt.edu; midkiff@vt.edu).

Y. Shi is with Intelligent Automation Inc., Rockville, MD, USA 20855 (email: yshi@vt.edu).

H. Zhou is with Wuhan Univ., Wuhan, Hubei, China 430072 (e-mail: bzhou@whu.edu.cn).

Please direct all correspondence to Prof. Tom Hou (thou@vt.edu), the Bradley Department of Electrical and Computer Engineering, 302 Whittemore Hall, MC 0111, Blacksburg, VA, USA 24061.

Color versions of one or more of the figures in this paper are available online at http://ieeexplore.ieee.org.

Digital Object Identifier 10.1109/JSAC.2015.2391631 and no line-of-sight (LOS) requirement [24]. Although wireless, WET does require the charging station to be brought to within reasonable range of a sensor node so that a good energy transfer efficiency can be achieved. We call this mobile vehicle platform (used to carry wireless charging station) as a wireless charging vehicle (WCV).

On the other hand, it has been well recognized that data collection with a mobile base station has significant advantages over a static one [13], [17], [26]. Given that a mobile base station also needs to be carried on a mobile platform, a natural question to ask becomes: Is it possible to dual use the WCV to carry the base station, in addition to its wireless charging station $?^{1}$ This is the main motivation of this investigation.

In this paper, we study the problem of employing a single mobile platform (the WCV) for both WET and data collection in a WSN. We assume the WCV follows a cyclic schedule its starts from its home service station, travels along a preplanned path and makes stops along the path for WET to sensor nodes, and returns to its home service station after it completes a tour. While traveling along its pre-planned path, where the WCV should stop and how long it will charge its neighboring sensor nodes are unknown and are part of our problem to solve. Further, it is necessary that data collection from all sensor nodes be relayed (via multi-hop) to the mobile base station (on WCV) in real time. That is, the multi-hop flow routing topology in the network is time-varying and is again part of our problem to solve. Apparently this is a very complex system, involving variables across multiple dimensions - time, space, and energy. The basic constraints in our problem are: (i) none of the sensor nodes run out of energy (so that data collection is never interrupted), and (ii) all data collected among the nodes is relayed to the base station in real time. The objective of our problem is to minimize energy consumption of the entire system. This objective will be elaborated in Section V.

Apparently, putting both WET and data collection on the same mobile platform is an interesting and challenging problem. The problem involves several subproblems, each of which is challenging on its own. First, the WCV's movement behavior needs to be optimized. Although the traveling path is given, where the WCV should make stops along this path and how long it should stay at each stop (for charging) remain to be solved. Second, the flow routing among sensor nodes need

\footnotetext{
${ }^{1}$ Although two separate mobile platforms may be employed (one for WCV and the other for mobile base station, the costs (both materials and energy) and algorithmic/protocol complexity associated with these two vehicles will be much higher.
} 
to be optimized, which depends on the location of WCV (and time). Finally, the WET efficiencies depends on the stopping locations and their distances to neighboring sensor nodes. Note that these subproblems are tightly coupled and requires a joint formulation and solution.

This paper addresses the above challenges by making the following contributions:

- We develop a mathematical model for the WCV as a mobile platform for both WET and data collection. This model consists of energy criteria to ensure that the energy level at each sensor node never falls below some minimum threshold, and a general optimization problem formulation (OPT-t) involving WCV's stopping behavior, energy charging, and data flow routing. For the objective function, we show that the goal of minimizing energy consumption associated with the WCV is equivalent to maximizing the fraction of the WCV's vacation time at the service station.

- Since OPT-t has a time-dependent formulation, it is difficult to develop a solution directly. Instead, we show that a special case of the OPT-t, where data flow routing and energy consumption of sensor nodes only depend on WCV's location, can offer the same optimal objective value as that for OPT-t. This finding allows us to shift our focus from OPT-t to this simpler formulation, denoted as OPT-s, which only involves location (space)-dependent variables.

- For problem OPT-s, we develop a $(1-\epsilon)$-optimal solution with any desired level of accuracy $\epsilon$. Our solution procedure involves discretizing traveling path into a finite number of segments and representing each segment as a logical point. By exploiting the worst case and the best case representation for each logical point, we can find a lower bound and an upper bound for OPT-s by solving two linear programs (LPs). Depending on the required accuracy, we show how to discretize segments into smaller segments. We prove that the gap between the lower and upper bounds closes as the number of iterations increases and we obtain a provably $(1-\epsilon)$ optimal solution.

It is worth pointing out that data collection process and charging process have inter-dependence on each other since both depend on the WCV's stopping behavior (i.e., where to stop on the pre-planned path and how long to stay at each stop). Therefore, the joint problem formulation in this paper cannot be decoupled into two separate problems.

The remainder of this paper is organized as follows. In Section II, we review related work on WET and mobile base station, respectively. In Section III, we give models for the WCV's stopping behavior, flow routing, energy charging and consumption in a WSN. Section IV presents WET criteria to ensure that each sensor node never runs out of energy. A general problem formulation (i.e., OPT-t) is given in Section V. In Section VI, we show that it is sufficient to study a special case of OPT-t that only involves space-dependent formulation, which we denote as OPT-s. Section VII presents a provably $(1-\epsilon)$-optimal solution to OPT-s. Section VIII presents numerical results and Section IX concludes this paper.

\section{RELATED WORK}

WET in Sensor Networks. Existing WET technologies can be classified into two categories: electromagnetic (EM) radiation and magnetic resonant coupling [24].

EM radiation transfers power via a radiative (far-field) electromagnetic field over a specified radio frequency (RF) band [14], [15]. In this frequency band, an RF transmitter emits radio waves, which are received by an RF receiver that tunes to this frequency band. In this manner, radio power can be harvested by the RF receiver. This technology has been applied to prolong lifetime of a WSN [3], [4], [5], [18]. However, there are a number of salient issues associated with this technology, such as extremely low energy transfer efficiency, requiring complicated tracking mechanisms if relative positions change, being sensitive to obstruction between the energy source and the receiver, and potential safety concerns.

Magnetic resonant coupling transfers energy from source to receiver by having a receiving coil operate at the same resonant frequency as the source coil via a nonradiative (nearfield) magnetic resonant induction [9], [10]. Compared to electromagnetic radiation, magnetic resonant coupling has the advantages of offering much higher energy transfer efficiency even under omni-direction, not requiring LOS, and being insensitive to the neighboring environment. Experiments in [9] showed that magnetic resonant coupling can transfer $60 \mathrm{~W}$ with $40 \%$ efficiency over a distance of 2 meters, which is simply not possible with EM radiation.

In [21], Xie et al. showed that by having a WCV visit and charge each sensor node in the network with this technology, a WSN can remain operational forever (i.e., infinite lifetime). In [22], Xie et al. addressed the scalability problem associated with WET in a dense sensor network by exploiting new advances in multi-node charging technology [10]. An assumption in [21], [22] was that the location of the base station is fixed. Mobile Base Station. The benefits of using a mobile base station for data collection in a sensor network have been well recognized in [1], [12], [13], [17], [27]. Due to the complexity associated with a mobile base station, early efforts either simplified the problem by limiting the locations of the base station to a finite set of points and assuming negligible traveling time between points [1], [13], or developed heuristic solutions under some simplified assumptions (e.g., a heuristic move of the base station toward sensor nodes with high traffic [27] or decoupling the base station's movement and data routing [12]). A major result on mobile base station research was given by Shi and Hou in [17], in which they showed a provably $(1-\epsilon)$-optimal solution. But it was assumed in [17] that the time for the base station to travel from one location to another is negligible. Further, there was no consideration of WET in [17].

It is important to contrast the mobile base station considered in this paper to data MULEs [16], message ferry [29], and SenCar [30]. The latter are employed in the so-called delay-tolerant network (DTN) [8], in which frequent network disconnectivity or partitioning is expected. Applications under 


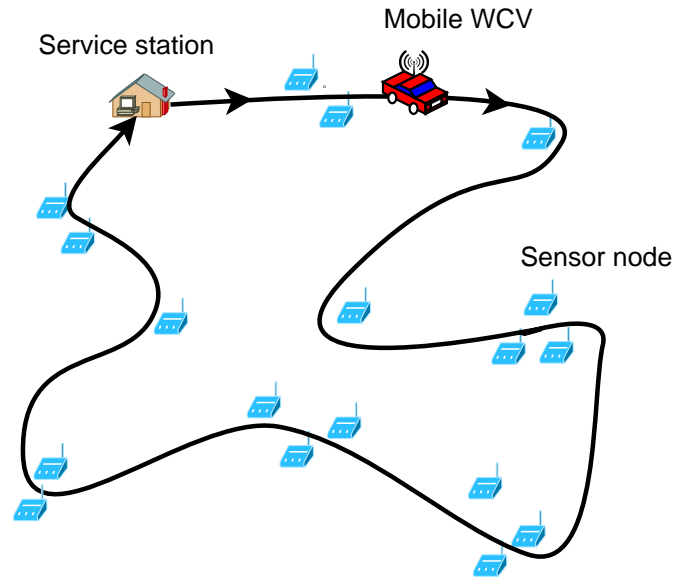

Fig. 1. An example WSN with a mobile WCV.

DTN are expected to tolerate large delays. In contrast, the mobile base station that we consider in this paper collects real-time data flow from sensor nodes with negligible delays. The mobile base station in this paper is also different from the mobile relay mechanism in [20], which employs a mobile node to help relay data from sensor nodes to a static base station.

\section{BASIC Network AND ENERGy MOdels}

In this section, we present some basic models for WCV's stopping behavior, flow routing, and energy consumption/reception in the WSN. Table I lists our notation.

\section{A. WCV and Travel Path}

Suppose we have a sensor network $\mathcal{N}$ deployed over a twodimensional area, with the location of each node $i \in \mathcal{N}$ being $\left(x_{i}, y_{i}\right)$. A WCV is employed to travel inside the network and charge the sensor nodes' batteries. This WCV starts from its home service station, travels along a pre-planned path in the area and returns to the service station at the end of its trip. While on its path, the WCV makes a number of stops and charges sensor nodes near those stops (see Fig. 1). In this paper, we assume that the WCV has sufficient amount of energy to support its travel, data collection, and energy transfer to sensor nodes before it returns to the service station. The detailed energy charging model will be described in Section III-D.

Denote $\mathcal{P}$ as the pre-planned traveling path and $\tau$ as the total amount of time for the WCV to complete a cycle. Then $\tau$ includes three components:

- The total travel time along the pre-planned path $\mathcal{P}$ is $D_{\mathcal{P}} / V$, where $D_{\mathcal{P}}$ is the distance along path $\mathcal{P}$ and $V$ is the traveling speed of the WCV.

- The vacation time $\tau_{\text {vac }}$, which refers the amount of time that the WCV remains at the service station (point $p_{\text {vac }}$ ) before it starts to travel.

- The total stopping (charging) time along the pre-planned path $\mathcal{P}$. Denote $\omega(p)$ as the aggregate amount of time the
TABLE I

NOTATION.

\begin{tabular}{|c|c|}
\hline$B$ & The base station \\
\hline$C_{i j}$ & $\begin{array}{l}\text { Energy consumption for transmitting one unit of } \\
\text { data rate from node } i \text { to node } j\end{array}$ \\
\hline $\begin{array}{c}C_{i B}(p) \\
\text { (or } C_{i B}(p(t)) \text { ) }\end{array}$ & $\begin{array}{l}\text { Energy consumption for transmitting one unit of } \\
\text { data rate from node } i \text { to the WCV at location } p \\
\text { (or time } t \text { ) }\end{array}$ \\
\hline$D_{i j}$ & Distance from node $i$ to node $j$ \\
\hline$D_{\delta}$ & Maximum charging distance of the WCV \\
\hline$D_{\mathcal{P}}$ & Distance over path $\mathcal{P}$ \\
\hline$E_{\max }$ & Full battery capacity at a sensor node \\
\hline$E_{\min }$ & $\begin{array}{l}\text { Minimum energy required in the battery to keep it } \\
\text { operational }\end{array}$ \\
\hline$e_{i}(t)$ & Energy level of sensor node $i$ 's battery at time $t$ \\
\hline $\begin{array}{c}f_{i j}(t) \\
\left.\text { (or } f_{i B}(t)\right)\end{array}$ & $\begin{array}{l}\text { Flow rate from sensor node } i \text { to sensor node } j \text { (or } \\
\text { base station } \mathrm{WCV} \text { ) at time } t\end{array}$ \\
\hline $\begin{array}{c}f_{i j}(p) \\
\left.\text { (or } f_{i B}(p)\right)\end{array}$ & $\begin{array}{l}\text { Flow rate from sensor node } i \text { to sensor node } j \text { (or } \\
\text { base station } \mathrm{WCV} \text { ) when the WCV is at location } p\end{array}$ \\
\hline $\begin{array}{c}G_{i} \\
M\left(\text { or } M_{i}\right)\end{array}$ & $\begin{array}{l}\text { Data rate generated at sensor node } i \\
\text { Number of segments (or number of segments at } i \text {-th } \\
\text { iteration) }\end{array}$ \\
\hline $\mathcal{N}$ & The set of sensor nodes in the network \\
\hline $\mathcal{P}$ & Traveling path of the WCV \\
\hline$p_{m}$ & $\begin{array}{l}\text { A logical point representing segment } \mathcal{S}_{m} \\
m=1,2, \ldots, M\end{array}$ \\
\hline$p_{\mathrm{vac}}$ & Location of the WCV's home service station \\
\hline$r_{i}(t)$ & Energy consumption rate at sensor node $i$ at time $t$ \\
\hline$r_{i}(p)$ & $\begin{array}{l}\text { Energy consumption rate at sensor node } i \text { when the } \\
\text { WCV is at location } p\end{array}$ \\
\hline $\mathcal{S}_{m}$ & $\begin{array}{l}\text { The } m \text {-th segment on path } \mathcal{P} \text {, which has been } \\
\text { discretized into } M \text { segments, } m=1, \ldots, M\end{array}$ \\
\hline$s(t)$ & $\begin{array}{l}\text { Distance traversed by the WCV on path } \mathcal{P} \text { up to } \\
\text { time } t\end{array}$ \\
\hline$U_{\max }$ & $\begin{array}{l}\text { Maximum output power from the WCV for a single } \\
\text { sensor node }\end{array}$ \\
\hline$U_{i B}(p)$ & Power reception rate at node $i$ when the WCV is at $p$ \\
\hline$V$ & Traveling speed of the WCV \\
\hline$\alpha$ & Path loss index \\
\hline$\beta_{1}$ & A constant in energy consumed for data transmission \\
\hline$\beta_{2}$ & $\begin{array}{l}\text { A coefficient in energy consumed for data } \\
\text { transmission }\end{array}$ \\
\hline$\rho$ & $\begin{array}{l}\text { Power consumption coefficient for receiving one unit } \\
\text { of data }\end{array}$ \\
\hline$\delta$ & A threshold of power reception rate \\
\hline$\epsilon$ & Targeted performance gap $(0<\epsilon \ll 1)$ \\
\hline$\eta_{\mathrm{vac}}$ & The ratio of the vacation time to the entire cycle time \\
\hline$\tau$ & Time spent to complete a cycle \\
\hline$T_{\mathcal{P}}$ & Time spent to travel along path $\mathcal{P}$ with speed $V$ \\
\hline$\tau_{\text {vac }}$ & $\begin{array}{l}\text { WCV's vacation time spent at its home service } \\
\text { station during a cycle }\end{array}$ \\
\hline$\omega(p)$ & $\begin{array}{l}\text { Aggregate time span for the WCV to stay at location } \\
p \in \mathcal{P}\end{array}$ \\
\hline$\mu(\cdot)$ & $\begin{array}{l}\text { The efficiency function of wireless energy transfer } \\
\text { from the WCV to a sensor node }\end{array}$ \\
\hline$\psi_{\text {OPT-t }}$ & A feasible solution (or an optimal solution) to \\
\hline$\left(\begin{array}{l}\left.\text { (or } \psi_{\text {OPT-t }}^{*}\right) \\
\psi_{\text {OPT- }}\end{array}\right.$ & $\begin{array}{l}\text { Problem OPT-t } \\
\text { A feasible solution (or an optimal solution) to }\end{array}$ \\
\hline$\left(\right.$ or $\psi_{\mathrm{OPT}-\mathrm{s}}^{*}$ & Problem OPT-s \\
\hline$\eta_{\mathrm{OPT}-\mathrm{s}}, \eta_{\mathrm{OPT}-\mathrm{s}}^{*}$ & The objective value achieved by $\psi_{\text {OPT-s }}$ and $\psi_{\text {OPT-s }}^{*}$ \\
\hline
\end{tabular}

WCV stops at point $p \in \mathcal{P}$. Since the WCV may stops at $p$ more than once during $\tau$, we have:

$$
\omega(p)=\int_{\{t \in[0, \tau]:(x, y)(t)=p\}} 1 \mathrm{~d} t,
$$

where $(x, y)(t)$ is the location of the WCV at time $t$. Then the total stopping time is $\sum_{p \in \mathcal{P}, p \neq p_{\text {vac }}}^{\omega(p)>0} \omega(p)$. 
Then we have:

$$
\tau=\frac{D_{\mathcal{P}}}{V}+\tau_{\text {vac }}+\sum_{p \in \mathcal{P}, p \neq p_{\text {vac }}}^{\omega(p)>0} \omega(p) .
$$

\section{B. Mobile Base Station and Data Flow Routing}

As discussed, the WCV is also serving as a mobile platform for the base station. Therefore, the base station is mobile while it acts as the sink node for all data collected from the sensor nodes in the network. In this paper, we assume a constant data generation rate from each sensor node, which is then relayed in real-time as data flow to the mobile base station. To conserve energy, multi-hop data routing is employed among the sensor nodes.

Suppose that each sensor node $i$ produces its local data with a constant rate $G_{i}$ (in b/s), $i \in \mathcal{N}$. Denote $f_{i j}(t)$ and $f_{i B}(t)$ as the flow rate from node $i$ to node $j$ and the base station at time $t$, respectively. Then we have the following flow balance at each sensor node $i$ :

$$
\sum_{k \in \mathcal{N}}^{k \neq i} f_{k i}(t)+G_{i}=\sum_{j \in \mathcal{N}}^{j \neq i} f_{i j}(t)+f_{i B}(t)(i \in \mathcal{N}, t \geq 0) .
$$

Note that we are modeling data collection as real-time fluid flow routing rather than delay-tolerant data delivery as in MULEs [16] or message ferry [29] type of communications.

\section{Sensor Energy Consumption}

At a sensor node, we assume communication activity is the dominant source of the node's energy consumption. ${ }^{2}$ We assume that a sensor node has power control capability so that each node can adjust its transmission power level based on its distance to the receive node. At the MAC layer, we assume a deterministic optimal scheduling where physicallayer interference can be effectively avoided. Denote $C_{i j}$ as the energy consumption rate for transmitting one unit of data flow from sensor node $i$ to sensor node $j$. Then $C_{i j}$ can be modeled as [2], [7]:

$$
C_{i j}=\beta_{1}+\beta_{2} D_{i j}^{\alpha},
$$

where $D_{i j}$ is the distance between nodes $i$ and $j, \beta_{1}$ and $\beta_{2}$ are constant terms, and $\alpha$ is the path loss index. Suppose that all sensor nodes are stationary, the inter-sensor distance $D_{i j}$ and $C_{i j}$ are all constants.

Denote $C_{i B}(p(t))$ as the energy consumption rate for transmitting one unit of data flow from sensor node $i$ to base station $B$ when $B$ is at location $p(t)$. Then for $i \in \mathcal{N}$, we have

$$
C_{i B}(p(t))=\beta_{1}+\beta_{2}\left[\sqrt{\left(x(t)-x_{i}\right)^{2}+\left(y(t)-y_{i}\right)^{2}}\right]^{\alpha},
$$

where $(x(t), y(t))$ and $\left(x_{i}, y_{i}\right)$ are the coordinates of $p(t)$ and node $i$, respectively. Note that unlike $C_{i j}$ 's, which are all constants, $C_{i B}(p(t))$ varies with the base station's position over time.

\footnotetext{
${ }^{2}$ Energy consumption for hardware device and information processing can be assumed to be constants and can be therefore easily integrated into total energy consumption if needed.
}

Denote $\rho$ as the rate of energy consumption for receiving one unit of data flow rate. Then the total energy consumption rate for both transmission and reception at node $i$, denoted as $r_{i}(t)$, is

$$
\begin{aligned}
r_{i}(t)=\rho \sum_{k \in \mathcal{N}}^{k \neq i} f_{k i}(t)+\sum_{j \in \mathcal{N}}^{j \neq i} C_{i j} \cdot f_{i j}(t) \\
+C_{i B}(p(t)) \cdot f_{i B}(t) \quad(i \in \mathcal{N}, t \geq 0) .
\end{aligned}
$$

\section{WET Charging Model}

In this paper, we adopt a multi-node, distance-dependent charging model. ${ }^{3}$ For simplicity, we assume that the WCV performs its charging function only when it stops at some point along the pre-planned path $\mathcal{P}$. Denote $U_{i B}(p)$ as the power reception rate at node $i$ when the $\mathrm{WCV}$ is located at $p \in \mathcal{P}$. Denote the efficiency of WET as $\mu\left(D_{i B}(p)\right)$, which is a decreasing function of distance $D_{i B}(p)$. Then the WET model is given as follows:

$$
U_{i B}(p)= \begin{cases}\mu\left(D_{i B}(p)\right) \cdot U_{\max } & \text { if } D_{i B}(p) \leq D_{\delta} \\ 0 & \text { if } D_{i B}(p)>D_{\delta},\end{cases}
$$

where $U_{\max }$ is the maximum output power for a single sensor node and $D_{\delta}$ is the charging range of the WCV, beyond which WET is not effective. In other words, $D_{\delta}$ is defined in such a way that the power reception rate at a sensor node is at least over a threshold value $\delta$. We assume that every sensor node is within a distance of $D_{\delta}$ to the path $\mathcal{P}$.

\section{ENERgy CyCles for SENSOR Nodes}

We assume the WCV follows a fixed travel schedule along pre-planned $\mathcal{P}$ with a period of $\tau$. Then we have $p(t)=p(t+$ $k \tau)$ for $0 \leq t \leq \tau, k=1,2, \cdots$. Further, we assume the flow routing in the network also follows a periodic cycle, i.e., $f_{i j}(t)=f_{i j}(t+k \tau)$ and $f_{i B}(t)=f_{i B}(t+k \tau)$ for $0 \leq t \leq \tau$, $k=1,2, \cdots$. Then by (5), the energy consumption rate at each node also follows a periodic cycle, i.e., $r_{i}(t)=r_{i}(t+k \tau)$ for $0 \leq t \leq \tau, i \in \mathcal{N}$. Since the WCV can charge its neighboring sensor nodes only when it stops, its charging behavior also follows a periodic cycle. In summary, the travel schedule of the WCV, WCV's charging behavior along its path, flow routing among the nodes, and energy consumption at each node are all periodic and repeats themselves for every period $\tau$.

Suppose that each sensor node has a battery capacity of $E_{\max }$ and is fully charged initially. Denote $E_{\min }$ as the minimum energy at a sensor node battery for it to be operational. We are interested in developing a particular travel cycle so that the energy at each sensor node at time $t$, denoted as $e_{i}(t)$, $i \in \mathcal{N}$, never falls below $E_{\min }$. In the following, we offer two constraints for the first cycle. We show that once these two constraints hold for the first cycle, then $e_{i}(t) \geq E_{\min }$ for $t \geq \tau$, i.e., the energy level at each sensor node never falls below $E_{\text {min }}$ for all future cycles.

\footnotetext{
${ }^{3}$ This model does not incorporate the effect of obstacles between a source coil and a receiver coil. It is a good abstraction for an obstacle-free environment.
} 
The first constraint ensures that $e_{i}(t)$, which starts from $E_{\max }$ at $t=0$, will not fall below $E_{\min }$ at $t=\tau$,

$$
E_{\max }-\left\{\int_{\{t \in[0, \tau]: \omega(p(t))=0\}} r_{i}(t) \mathrm{d} t+\right.
$$

$\left.\int_{\left\{t \in[0, \tau]: \omega(p(t))>0, D_{i B}(p(t))>D_{\delta}\right\}} r_{i}(t) \mathrm{d} t\right\} \geq E_{\min } \quad(i \in \mathcal{N}),(7)$

where $\int_{\{t \in[0, \tau]: \omega(p(t))=0\}} r_{i}(t) \mathrm{d} t$ is the amount of energy consumed at node $i$ when the WCV is moving along path $\mathcal{P}$ while $\int_{\left\{t \in[0, \tau]: \omega(p(t))>0, D_{i B}(p(t))>D_{\delta}\right\}} r_{i}(t) \mathrm{d} t$ is the amount of energy consumed at node $i$ when the WCV is making stops to charge other nodes (not including $i$ in its charging range).

The second constraint ensures that $e_{i}(t)$, which starts from $E_{\max }$ at $t=0$, will be charged back to $E_{\max }$ before the end of the first cycle $\tau$. We have

$\int_{0}^{\tau} r_{i}(t) \mathrm{d} t \leq \sum_{p \in \mathcal{P}}^{\omega(p)>0, D_{i B}(p) \leq D_{\delta}} U_{i B}(p) \cdot \omega(p) \quad(i \in \mathcal{N}),(8)$

where the left hand side is the amount of energy consumed at node $i$ during $\tau$ and the right hand side is the maximum possible amount of energy received by node $i$ in a cycle. Note that the actual amount of energy received by node $i$ in the first cycle may be less than the right hand side due to battery overflow. ${ }^{4}$

Note that (7) and (8) characterize the energy consumption and reception in the first cycle. The following lemma says that if both (7) and (8) hold for the first cycle, then we have $e(t) \geq E_{\min }$ for all subsequent cycles.

Lemma 1 (Energy Criteria): If both (7) and (8) are satisfied for the first cycle, then $e_{i}(t) \geq E_{\text {min }}$ for all $t \geq 0, i \in \mathcal{N}$.

A proof sketch: To prove Lemma 1, we consider the first cycle and the subsequent cycles, separately. The first cycle (i.e., $t \in[0, \tau]$ ) is considered separately since it starts with $E_{\max }$, and the subsequent cycles (i.e., $t \in[k \tau,(k+1) \tau]$, $k \in \mathbb{N}^{+}$) may start with a energy level lower than $E_{\max }$. For the subsequent cycles, we can show that $e_{i}(t) \geq E_{\min }$ for the second cycle and a node's energy behavior repeats from cycle to cycle. To show that $e_{i}(t) \geq E_{\min }, t \in[\tau, 2 \tau], i \in \mathcal{N}$, we need to show that node $i$ can be charged back to $E_{\max }$ in the first and the second cycles. Based on this result, we can further exploit the cyclic behavior of traveling, energy charging and energy consumption among the cycles. Since $e_{i}(t) \geq E_{\min }$ for the first cycle, we can have $e_{i}(t) \geq E_{\min }$ for the second cycle. Then, we can show that $e_{i}(2 \tau)=e_{i}(\tau)$, which yields $e_{i}(t) \geq E_{\min }$ for all subsequent cycles due to periodicity. A formal proof of Lemma 1 is given in [23].

\section{Problem Formulation}

In Section IV, we discussed constraints to form energy cycles at a sensor node. Under Lemma 1 , we showed that under certain conditions, $e_{i}(t) \geq E_{\min }$ for all $t \geq 0, i \in \mathcal{N}$. Based on these constraints, we would like to optimize some global performance objective. In particular, we want to minimize the rate of energy consumption for the entire system, including: ${ }^{5}$

\footnotetext{
${ }^{4}$ Once a battery is charged to $E_{\max }$, its energy level cannot be further increased.

${ }^{5}$ Note that except its initial energy, the energy consumed by each sensor node in the WSN comes from the WCV.
}

- The energy consumption rate for carrying the WCV to move along the pre-planned path $\mathcal{P}$ during $\tau$ :

$$
\frac{R_{v} \cdot\left[\frac{D_{\mathcal{P}}}{V}+\sum_{p \in \mathcal{P}, p \neq p_{\text {vac }}}^{\omega(p)>0} \omega(p)\right]}{\tau},
$$

where $D_{\mathcal{P}} / V+\sum_{p \in \mathcal{P}, p \neq p_{\text {vac }}}^{\omega(p)>0} \omega(p)$ is the time that the $\mathrm{WCV}$ is at work during $\tau$ (i.e., outside its service station), and $R_{v}$ denotes the average energy consumption rate by the mobile vehicle in this period. Note that the energy consumed by the mobile vehicle when it makes stops along $\mathcal{P}$ should be included as the vehicle's engine is still on.

- The rate of energy transferred from the WCV to the sensor nodes in the network is at most $\frac{\sum_{i \in \mathcal{N}} r_{i}}{\delta / U_{\max }}$, where $\sum_{i \in \mathcal{N}} r_{i}$ is the sum of energy consumption rates at the sensor nodes, and $\delta / U_{\max }$ is the minimum efficiency for effective wireless charging. ${ }^{6}$

To date, the most efficient electric passenger vehicle has an average energy consumption of $17.4 \mathrm{~kW}-\mathrm{h}$ per $100 \mathrm{~km} \mathrm{[19],}$ which indicates that $R_{v}$ is on the order of $1 \mathrm{~kW} .^{7}$ In contrast, the rate of energy transferred to sensor nodes is roughly on the order of 0.1 to $1 \mathrm{~W}$ (see Section VIII). Since the energy consumed by the mobile vehicle is the dominant source of energy consumption, we only need to focus on this dominant part, i.e., minimizing (9). Since $R_{v}$ is a constant factor, we can drop it from the objective function.

It is interesting that, by (2), minimizing $\frac{D_{\mathcal{P}} / V+\sum_{p \in \mathcal{P}, p \neq p_{\text {vac }}}^{\omega(p)>0} \omega(p)}{\tau}$ is equivalent to maximizing $\frac{\tau_{\text {vac }}}{\tau}$, which is the percentage of time that the $\mathrm{WCV}$ is on vacation at its service station. Therefore, we have the following optimization problem:

\section{OPT-t:}

maximize $\frac{\tau_{\mathrm{vac}}}{\tau}$

s.t. Time constraints: (1), (2);

Flow routing constraints: (3);

Energy consumption model: (4), (5);

Energy criteria constraints: (7), (8).

$\tau, \tau_{\text {vac }}, \omega(p) \geq 0,(x, y)(t) \in \mathcal{P} \quad(p \in \mathcal{P}, 0 \leq t \leq \tau)$ $f_{i j}(t), f_{i B}(t), C_{i B}(t), r_{i}(t) \geq 0,(i, j \in \mathcal{N}, i \neq j, 0 \leq t \leq \tau)$.

In this formulation, $\mathcal{P}, D_{\mathcal{P}}, V, G_{i}, \beta_{1}, \beta_{2}, \alpha, x_{i}, y_{i}, \rho$, $C_{i j}, E_{\max }$, and $E_{\min }$ are given a priori, and $U_{i B}(p)$ can be computed by (6). The time intervals $\tau$, $\tau_{\text {vac }}$, and $\omega(p)$, the location $(x, y)(t)$, the flow rates $f_{i j}(t)$ and $f_{i B}(t)$, the unit cost rate $C_{i B}(t)$, and the power consumption rate $r_{i}(t)$ are optimization variables. Among these variables, there are three sets of variables that constitute the solution space: (i) the WCV's location (i.e., $(x, y)(t)$ ); (ii) the WCV's sojourn time at each location $p \in \mathcal{P}$ and $p \neq p_{\text {vac }}$ (i.e., $\omega(p)$ ) or vacation time at the service station (i.e., $\tau_{\text {vac }}$; (iii) the corresponding

\footnotetext{
${ }^{6}$ From experimental results in [10], the ratio of $\delta$ to $U_{\max }$ can be set as $20 \%$ (see Section VIII).

${ }^{7}$ Note that this number was taken from data for an electric passenger vehicle with a maximum speed of $100+\mathrm{km} / \mathrm{h}$. For wireless charging in a sensor network, a much smaller autonomous vehicle traveling at a much lower speed and consuming much less energy would be enough, although we do not have exact data available in the literature.
} 


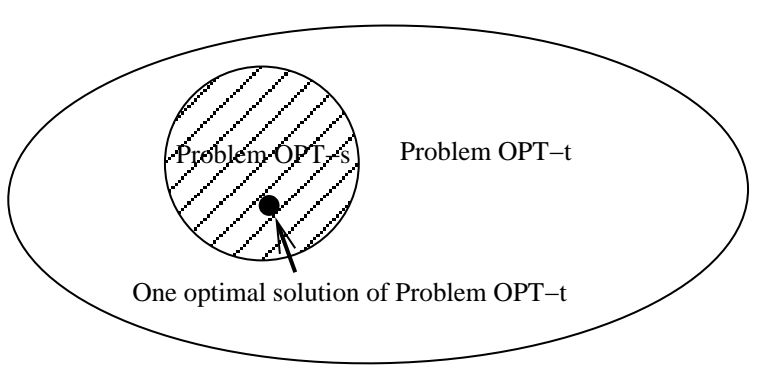

Fig. 2. Solution space for problems OPT-t and OPT-s.

flow routing (i.e., $f_{i j}(t)$ and $f_{i B}(t)$ ). Problem OPT-t exhibits a continuous-time nonlinear program [28], and is NP-hard in general.

\section{Downsizing Solution Space: A Location-Based FORMULATION}

Roadmap. OPT-t is a general formulation of our problem. It is difficult to solve as its variables are time-dependent (e.g., $\left.(x, y)(t), f_{i j}(t)\right)$. In this general formulation, OPT-t allows that data flow routing and energy consumption of sensor nodes vary over time, even when the WCV visits the same location.

In this section, we consider a special case of problem OPT-t, where data flow routing and energy consumption of sensor nodes only depend on the WCV's location. That is, regardless when the WCV visits a location $p \in \mathcal{P}$, the data flow routing and energy consumption of sensor nodes are the same as long as the WCV is at this location. We call this special case of problem OPT-t as location (space)-dependent problem and denote it as OPT-s. The solution space for OPT-s and OPT-t is shown in Fig. 2, where solution space for OPT-s is completely contained in OPT-t.

We now show that the optimal objective value for OPT-s is the same as that for OPT-t, despite that its solution space is smaller (Theorem 1). This result is significant as it allows us to study OPT-s, which is a subproblem of OPT-t and has a simpler formulation that just involves location-dependent variables.

Location-dependent Formulation. We now formulate OPTs. First, we need some new notation. Denote $f_{i j}(p)$ and $f_{i B}(p)$ as flow rates from sensor node $i$ to sensor node $j$ and to the base station when the WCV is at location $p \in \mathcal{P}$, respectively. Then (3) is rewritten as

$$
\sum_{k \in \mathcal{N}}^{k \neq i} f_{k i}(p)+G_{i}=\sum_{j \in \mathcal{N}}^{j \neq i} f_{i j}(p)+f_{i B}(p)(i \in \mathcal{N}, p \in \mathcal{P}) \text {. }
$$

Similarly, denote $C_{i B}(p)$ and $r_{i}(p)$ as the energy consumption for transmitting one unit of data rate from node $i$ to the base station and the energy consumption rate at node $i$ when the WCV is at location $p \in \mathcal{P}$, respectively. Then (5) can be rewritten as:

$$
\begin{aligned}
r_{i}(p)=\rho \sum_{k \in \mathcal{N}}^{k \neq i} f_{k i}(p)+\sum_{j \in \mathcal{N}}^{j \neq i} C_{i j} \cdot f_{i j}(p) \\
+C_{i B}(p) \cdot f_{i B}(p) \quad(i \in \mathcal{N}, p \in \mathcal{P}) .
\end{aligned}
$$

Now we rewrite (7) and (8) into location-based constraints. We start with (8). In (8), $\int_{0}^{\tau} r_{i}(t) \mathrm{d} t$ can be split into two parts:
- Energy consumed when the WCV makes stops (including vacation at the service station), which is, $r_{i}\left(p_{\text {vac }}\right) \cdot \tau_{\text {vac }}+$ $\sum_{p \in \mathcal{P}, p \neq p_{\text {vac }}}^{\omega(p)>0} r_{i}(p) \cdot \omega(p)$;

- Energy consumed when WCV travels along $\mathcal{P}$, i.e.,

$$
\int_{t \in[0, \tau]}^{\omega(p(t))=0} r_{i}(t) \mathrm{d} t=\int_{s \in\left[0, D_{\mathrm{P}}\right]}^{\omega(p(s))=0} r_{i}(p(s)) \frac{d t}{d s} \mathrm{~d} s,
$$

where $s \in\left[0, D_{\mathcal{P}}\right]$ is the distance traversed by the WCV along $\mathcal{P}$ (starting from its service station), and $p(s)$ is the WCV's location corresponding to $s$. Denote $W=$ $\lim _{\Delta \rightarrow 0} \frac{\Delta t}{\Delta s}$. Then $W=\frac{1}{V}$. Thus, (12) can be rewritten as:

$$
\int_{t \in[0, \tau]}^{\omega(p(t))=0} r_{i}(t) \mathrm{d} t=\int_{s \in\left[0, D_{\mathrm{P}}\right]}^{\omega(p(s))=0} W \cdot r_{i}(p(s)) \mathrm{d} s .
$$

Based on the above discussion, (8) can be rewritten as :

$$
\begin{aligned}
& r_{i}\left(p_{\text {vac }}\right) \cdot \tau_{\text {vac }}+\sum_{p \in \mathcal{P}, p \neq p_{\text {vac }}}^{\omega(p)>0} r_{i}(p) \cdot \omega(p) \\
&+\int_{s \in\left[0, D_{\mathrm{P}}\right]}^{\omega(p(s))=0} W \cdot r_{i}(p(s)) \mathrm{d} s \\
& \leq \sum_{p \in \mathcal{P}}^{\omega(p)>0, D_{i B}(p) \leq D_{\delta}} U_{i B}(p) \cdot \omega(p),(i \in \mathcal{N}),
\end{aligned}
$$

which is a location-dependent formulation. Similarly, (7) can be rewritten as follows:

$$
\begin{gathered}
r_{i}\left(p_{\text {vac }}\right) \cdot \tau_{\text {vac }}+\sum_{p \in \mathcal{P}, p \neq p_{\text {vac }}}^{\omega(p)>0, D_{i}(p)>D_{\delta}} r_{i}(p) \cdot \omega(p) \\
+\int_{s \in\left[0, D_{\mathrm{P}}\right]}^{\omega(p(s))=0} W \cdot r_{i}(p(s)) \mathrm{d} s \leq E_{\text {max }}-E_{\text {min }} \quad(i \in \mathcal{N}) .
\end{gathered}
$$

We now have a formulation for OPT-s, which only involves location-dependent variables.

\section{OPT-s:}

maximize $\frac{\tau_{\mathrm{vac}}}{\tau}$

s.t. Time constraints: (2);

Flow routing constraints: (10);

Energy consumption model: (11);

Energy criteria constraints: (13), (14).

$\tau, \tau_{\text {vac }}, \omega(p) \geq 0 \quad(p \in \mathcal{P})$

$f_{i j}(p), f_{i B}(p), r_{i}(p) \geq 0 \quad(i, j \in \mathcal{N}, i \neq j, p \in \mathcal{P})$.

Proof of Equivalence. We now show that the optimal objective value of OPT-s is the same as that for OPT-s. For Problem OPT-t, denote $\psi_{\text {OPT-t }}$ and $\psi_{\text {OPT-t }}^{*}$ as a feasible solution and an optimal solution, respectively. Similarly, for Problem OPT-s, denote $\psi_{\text {OPT-s }}$ and $\psi_{\text {OPT.s }}^{*}$ as a feasible solution and an optimal solution, respectively.

Theorem 1: The optimal objectives by $\psi_{\text {OPT-S }}^{*}$ and $\psi_{\text {OPT-t }}^{*}$ are identical.

The proof of this theorem is based on two results. First, we show the optimal objective of $\psi_{\text {oPT-t }}^{*}$ is no less than that in solution $\psi_{\text {OPT.s }}^{*}$. This is straightforward as OPT-s is a subproblem of OPT-t. Second, we show that the converse is also true. Instead of considering an optimal solution, we will show that for any objective value achieved by a feasible solution $\psi_{\text {OPT-t }}$, we can always find a feasible solution $\psi_{\text {OPT-s }}$ that has the same objective value (Lemma 2). If this is true, in the special case when the feasible solution $\psi_{\text {opt-t }}$ is the optimal solution $\psi_{\text {OPT-t }}^{*}$, the objective by $\psi_{\text {OPT-t }}^{*}$ can also be achieved by some feasible solution $\psi_{\text {opts. }}$. 


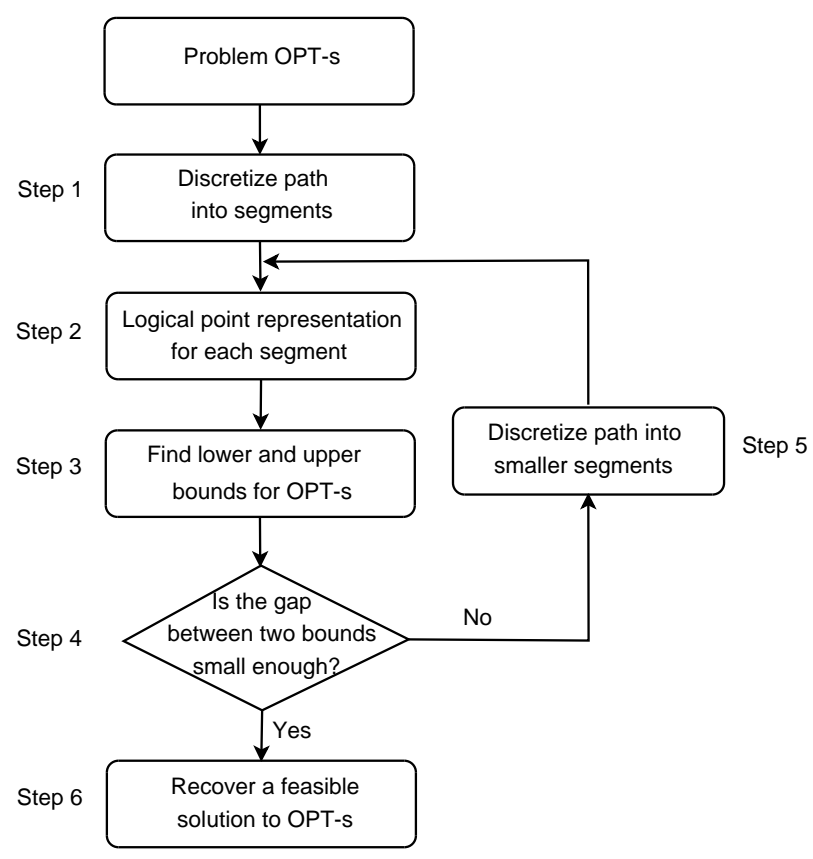

Fig. 3. A flow-chart of our algorithm.

Lemma 2: Given a feasible $\psi_{\text {OPT- }}$, we can construct a feasible $\psi_{\text {opts }}$ with the same objective value.

A formal proof of Lemma 2 is given in the appendix. Based on Theorem 1, we conclude that as far as our objective is concerned, it is sufficient to study OPT-s, which has a simpler formulation than OPT-t.

\section{A NeAR-Optimal Solution to Problem OPT-S}

Although OPT-s is simpler than OPT-t, path $\mathcal{P}$ still has infinite number of points. In this section, by discretizing path $\mathcal{P}$ into a finite number of segments and representing each segment as a logical point, we develop a provably $(1-\epsilon)$ near-optimal solution.

\section{A. Basic Idea}

Fig. 3 shows the flow chart of our proposed algorithm. In Step 1, we discretize path $\mathcal{P}$ into $M_{1}$ segments of equal length, i.e., $D_{\mathcal{P}} / M_{1}$. For each segment, we represent it as a logical point. Once the WCV is within a segment, we say that it is visiting the corresponding logical point, regardless the specific location within this segment. Note that the time that the WCV spends at a logical point includes both its traveling time on this segment as well as stopping time at any point in this segment for charging.

Since each sensor node's energy consumption and charging behavior depend on the specific location of the WCV within a segment, it is not obvious how we should characterize its energy behavior for the corresponding logical point. Fortunately, we find that for the purpose of developing a $(1-\epsilon)$-optimal algorithm, it is sufficient to use the worst case behavior. That is, to characterize a sensor node's energy consumption and charging behavior when the WCV is at a logical point, it is sufficient to consider maximum energy consumption and minimum energy reception.
Once we have a worst case representation for each logical point, in Step 3, we can find a lower bound for OPT-s by solving a LP. Also in Step 3, by developing a best case representation for each logical point (following the same token for the worst case representation), we can find an upper bound for OPT-s by solving another LP.

In Step 4, we check the gap between objective values from the lower and upper bounds. If the gap is within $\epsilon$ of the upper bound, we have a $(1-\epsilon)$-optimal solution (corresponding to the lower bound solution, which is feasible); otherwise, we increase the number of segments (Step 5) linearly and return to Step 2.

As the iteration continues, we expect the gap between lower and upper bounds get closer and closer and we shall have a $(1-\epsilon)$ solution (when the lower bound is within $(1-\epsilon)$ of upper bound). From the solution corresponding to the final lower bound, we can construct a feasible solution to Problem OPT-s (Step 6).

In the remainder of this section, we give details of each step of our algorithm, prove its $(1-\epsilon)$ optimality and discuss algorithm's complexity.

\section{B. Path Discretization (Step 1) and Logical Point Represen- tation (Step 2)}

In this section, we elaborate how to discretize the traveling path into a finite number of segments, and represent each segment as a logical point.

Path Discretization (Step 1). In the first iteration, path $\mathcal{P}$ is equally divided into $M_{1}$ segments, each of which is indexed in increasing order following the WCV's traveling direction. Denote $\mathcal{S}_{m}$ and $D\left(\mathcal{S}_{m}\right), m=1,2, \ldots, M_{1}$, as the $m$-th segment and its length, with $D\left(\mathcal{S}_{m}\right)=D_{\mathcal{P}} / M_{1}$, $m=1,2, \ldots, M_{1}$.

Logical Point Representation (Step 2). For segment $\mathcal{S}_{m}, m=1,2, \ldots, M_{1}$, we represent it as a logical point $p_{m}$. The traveling time spent by the WCV on segment $\mathcal{S}_{m}$ (excluding stopping time) is $D\left(\mathcal{S}_{m}\right) / V$. Denote $\omega\left(p_{m}\right)$ as the total stopping time that the WCV is in segment $\mathcal{S}_{m}$, i.e., $\omega\left(p_{m}\right)=\sum_{p \in \mathcal{S}_{m}}^{\omega(p)>0} \omega(p)$. Denote $\tau\left(p_{m}\right)$ as the total time that the WCV spends at $p_{m}$, which includes traveling time and stopping time. Then,

$$
\tau\left(p_{m}\right)=\frac{D\left(\mathcal{S}_{m}\right)}{V}+\sum_{p \in \mathcal{S}_{m}}^{\omega(p)>0} \omega(p) .
$$

Note that energy consumption rate $C_{i B}(p)$ and energy reception rate $U_{i B}(p)$ may vary at different point in the same segment. To develop a $(1-\epsilon)$-optimal solution, we use the worst case values of energy consumption rate and energy reception rate within the segment to characterize a logical point. That is, for $i \in \mathcal{N}$, define $U_{i B}\left(p_{m}\right)$ and $C_{i B}\left(p_{m}\right)$ as

$$
\begin{aligned}
& U_{i B}\left(p_{m}\right) \triangleq \min _{p \in \mathcal{S}_{m}}\left\{U_{i B}(p)\right\}, \\
& C_{i B}\left(p_{m}\right) \triangleq \max _{p \in \mathcal{S}_{m}}\left\{C_{i B}(p)\right\} .
\end{aligned}
$$

Since there are $|\mathcal{N}|$ sensor nodes in the network, a logical point $p_{m}$ is represented by $2|\mathcal{N}|$-tuple vector $\left[U_{1 B}\left(p_{m}\right)\right.$, $\left.\ldots, U_{|\mathcal{N}| B}\left(p_{m}\right), C_{1 B}\left(p_{m}\right), \ldots, C_{|\mathcal{N}| B}\left(p_{m}\right)\right]$. 


\section{Find Lower and Upper Bounds (Step 3)}

Once we have a worst case representation for each logical point, we can obtain a formulation for a lower bound to OPT-s, which we denote as OPT-lb. Through a systematic change-ofvariable technique, OPT-lb can be reformulated into an LP and can be solved in polynomial time (see [23] for more details).

Following the same token, we can find an upper bound to OPT-s by developing a best case representation for each logical point. This is done by defining

$$
\bar{U}_{i B}\left(p_{m}\right) \triangleq \max _{p \in p_{m}}\left\{U_{i B}(p)\right\}, \bar{C}_{i B}\left(p_{m}\right) \triangleq \min _{p \in \mathcal{S}_{m}}\left\{C_{i B}(p)\right\} .
$$

Based on this best-case representation, we obtain a formulation for an upper bound to OPT-s, denoted as OPT-ub. Similar to OPT-lb, OPT-ub can be reformulated into an LP and can be solved in polynomial time.

\section{Termination Condition (Step 4) and Further Discretization (Step 5)}

We can check the gap between the lower and upper bounds. If the gap is within desired accuracy (denoted by $\epsilon$ ), the algorithm terminates and returns a $(1-\epsilon)$-optimal solution; otherwise, we further discretize existing segments into smaller ones and perform another iteration and so forth.

For discretization, we denote $M_{i}, i=1,2, \cdots$, as the number of segments in the $i$-th iteration. By intuition, among all variables, $\omega\left(p_{m}\right)$ 's directly determine the WCV's stopping behavior at $p_{m}$ and thus the final objective value. Thus, we sort segments $p_{m}, m=1,2, \ldots, M_{i}$, in the decreasing value of $\omega\left(p_{m}\right)$ (a tie is broken randomly). From the ordered segments, we pick the first $|\mathcal{N}|$ segments and equally subdivide each of them into two segments. ${ }^{8}$ As a result, at most $|\mathcal{N}|$ segments are added at each iteration, i.e., a linear increase in the number of segments.

Denote $\psi_{\text {OPT-lb }}^{\left(M_{i}\right)}$ and $\psi_{\text {OPT-ub }}^{\left(M_{i}\right)}$ as the optimal solutions to OPT-lb and OPT-ub at the end of the $i$-th iteration, respectively. Denote $\eta_{\mathrm{vac}}^{\left(M_{i}\right)}$ and $\bar{\eta}_{\mathrm{vac}}^{\left(M_{i}\right)}$ as the objective values in $\psi_{\mathrm{OPT}-\mathrm{lb}}^{\left(M_{i}\right)}$ and $\psi_{\mathrm{OPT}-\mathrm{ub}}^{\left(M_{i}\right)}$, respectively. Then we have the following lemma:

Lemma 3: For $i \geq 1, \eta_{v a c}^{\left(M_{i+1}\right)}>\eta_{v a c}^{\left(M_{i}\right)}$, and $\bar{\eta}_{v a c}^{\left(M_{i+1}\right)}<$ $\bar{\eta}_{v a c}^{\left(M_{i}\right)}$.

Lemma 3 says that as the number of segments increases with the number of iterations, the lower bound to OPT-s strictly increases while the upper bound strictly decreases. Lemma 3 can be proved by construction, and we give a proof sketch as follows. By a finer path segmentation, we can always construct an improved solution (in terms of objective function value) for the lower bound to OPT-s. The basic idea is that dividing a segment into smaller pieces can offer improved worst case bound (on energy consumption and/or energy charging), which leads to less charging time (than that in the coarser segmentation) and increased objective function value. Similarly, we can show that for the upper bound to OPT-s, the solution gets worse with a finer path segmentation. Therefore, as the number of segments increases with the

\footnotetext{
${ }^{8}$ As there are $|\mathcal{N}|$ sensor nodes, the number of stops by the WCV (for charging) should be on the same order of $|\mathcal{N}|$. If $M_{i}<|\mathcal{N}|$, then we just divide all $M_{i}$ segments.
}

number of iterations, we have a strictly increasing lower bound to OPT-s and a strictly decreasing upper bound. A complete proof is given in [23].

\section{E. Recover a Feasible Solution to OPT-s (Step 6)}

Once the lower bound is within $(1-\epsilon)$ of the upper bound, the algorithm terminates. With the current lower bound solution to OPT-lb (denoted as $\psi_{\text {OPT-1b }}$ ), we can construct a feasible solution to OPT-s (denoted as $\psi_{\text {OPT-s }}$ ). Solution $\psi_{\text {oPT-s }}$ consists of several components, including (i) cycle time $\tau$; (ii) for $p \in \mathcal{P}, p \neq p_{\text {vac }}$, stopping time $\omega(p)$, flow routing $f_{i j}(p)$ and $f_{i B}(p)$, and energy consumption rate $r_{i}(p)$; and (iii) vacation time at the service station $\tau_{\text {vac }}$, and corresponding flow routing $f_{i j}\left(p_{\text {vac }}\right)$ and $f_{i B}\left(p_{\text {vac }}\right)$, and energy consumption rate $r_{i}\left(p_{\text {vac }}\right)$. From $\psi_{\text {OPT-lb }}, \psi_{\text {OPT-s }}$ can be constructed as follows:

- It has the same cycle time $\tau$ as that in $\psi_{\text {oPT-1b }}$.

- For $p \in \mathcal{P}$ but $p \neq p_{\text {vac }}$, the WCV may traverse segment $\mathcal{S}_{m}$ with or without any stop. To see if the WCV makes any stop in $\mathcal{S}_{m}$, we calculate $\tau\left(p_{m}\right)-D\left(\mathcal{S}_{m}\right) / V$. If the difference is greater than 0 , then the WCV stops within $\mathcal{S}_{m}$ and in $\psi_{\text {oPT-s }}$ we may choose any point $p \in \mathcal{S}_{m}$. For this $p$, the WCV will stay for $\tau\left(p_{m}\right)-D\left(\mathcal{S}_{m}\right) / V$ amount of time. Otherwise (i.e., $\tau\left(p_{m}\right)-D\left(\mathcal{S}_{m}\right) / V=0$ ), the WCV does not stop within $\mathcal{S}_{m}$. Regardless of stopping or not, the flow routing solution at a point $p \in \mathcal{S}_{m}$ is: $f_{i j}(p)=f_{i j}\left(p_{m}\right)$ and $f_{i B}(p)=f_{i B}\left(p_{m}\right)$. Corresponding to $p \in \mathcal{S}_{m}, r_{i}(p)$ is defined by (11).

- For $p=p_{\text {vac }}$, we have that $\tau_{\text {vac }}, f_{i j}\left(p_{\text {vac }}\right), f_{i B}\left(p_{\text {vac }}\right)$, and $r_{i}\left(p_{\text {vac }}\right)$ are the same as those in $\psi_{\text {oPT-lb }}$.

Denote $\eta_{\text {OPT-lb }}$ and $\eta_{\text {OPT-s }}$ as the objective values achieved by $\psi_{\text {OPT-lb }}$ and $\psi_{\text {OPT-s }}$, respectively. Since $\tau$ and $\tau_{\text {vac }}$ are unchanged in the foregoing solution construction, we have

$$
\eta_{\text {OPT-s }}=\eta_{\text {OPT-Ib }} \text {. }
$$

\section{Lemma 4: $\psi_{\text {OPT.s }}$ is feasible to Problem OPT-s.}

A proof of Lemma 4 is based on the fact that OPT-lb is a worst case representation of OPT-s. By (15), we have $U_{i B}\left(p_{m}\right) \leq$ $U_{i B}(p)$, and by (16), we have $C_{i B}\left(p_{m}\right) \geq C_{i B}(p), p \in \mathcal{S}_{m}$. In other words, a logical point in OPT-lb is subject to stricter requirements (in term of energy charging and consumption) than any physical point within the corresponding segment in OPT-s. Thus, if a solution meets such stricter requirements enforced by a logical point, it must meet the looser requirements by its physical points, which yields a feasible solution to Problem OPT-s. A complete proof of Lemma 4 is given in [23].

\section{F. Proof of $(1-\epsilon)$ Optimality and Algorithm Complexity}

Let $\eta_{\mathrm{OPT} \text {-s }}^{*}$ be the (unknown) optimal objective value to OPTs. The following theorem says that the constructed solution $\psi_{\mathrm{OPT} \text { s }}$ is $(1-\epsilon)$-optimal.

Theorem 2: For any $0<\epsilon \ll 1, \eta_{\text {OPT.S }} \geq(1-\epsilon) \eta_{\text {OPT.S }}^{*}$.

Proof: Upon termination, we have $\eta_{\text {oPT-lb }} \geq(1-\epsilon) \eta_{\text {OPT-ub }}$, where $\eta_{\text {OPT-lb }}$ and $\eta_{\text {OPT-ub }}$ are the objective values achieved by the 


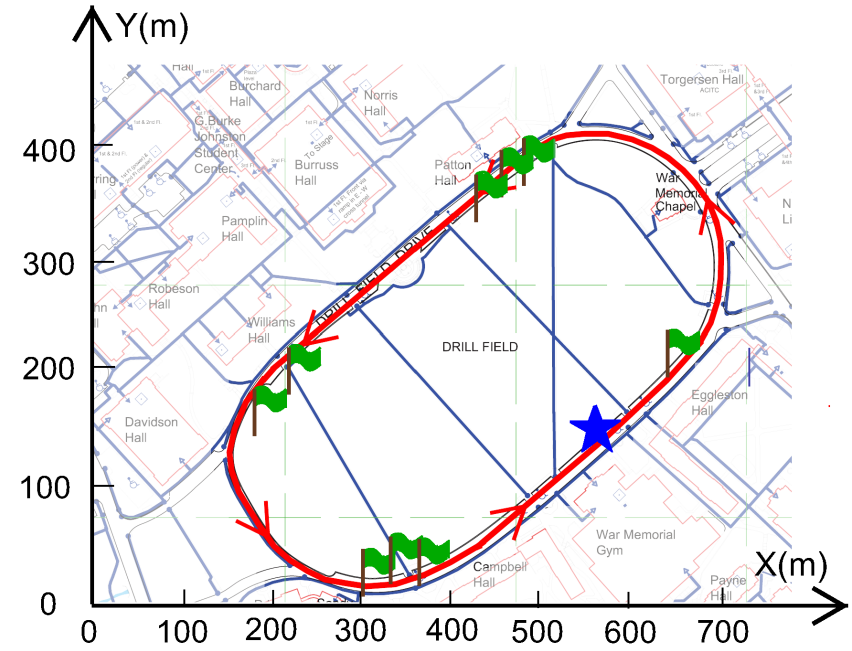

Fig. 4. Drillfield driveway in Virginia Tech campus. The solid line with arrows represent the WCV's traveling path. A star represents the WCV's home service station. A flag represents a stopping point in the case study (Section VIII-B).

lower bound solution to OPT-lb and the upper bound solution to OPT-ub, respectively. Therefore, we have

$$
\eta_{\mathrm{OPT}-\mathrm{s}}=\eta_{\mathrm{OPT}-\mathrm{lb}} \geq(1-\epsilon) \eta_{\mathrm{OPT}-\mathrm{ub}} \geq(1-\epsilon) \eta_{\mathrm{OPT}-\mathrm{s}}^{*},
$$

where the first equality holds by (17), and the last inequality holds since $\eta_{\mathrm{OPT}-\mathrm{ub}}$ is an upper bound for OPT-s. This completes the proof.

Regarding algorithmic complexity, each iteration in the algorithm solves two LPs (in Step 3), which can be done in polynomial time. Since at most $|\mathcal{N}|$ new segments are added at the end of each iteration, the number of segments as well as the problem size increases linearly. Although we do not have a worst case bound for the maximum number of iterations, it turns out that the convergence to optimality is extremely fast (typically only a few iterations) in practice (see Section VIII).

\section{NUMERICAL RESULTS}

In this section, we use numerical results to demonstrate how our algorithm solves the OPT-s problem.

\section{A. Network and Parameter Settings}

We use Virginia Tech's Drillfield (see Fig. 4) for sensor network deployment. Sensor nodes are deployed within a distance of the charging range along the side of the Drillfield driveway, which is roughly an ellipse. The home service station (marked as a star in Fig. 4) is located at $(540,160)$ (in m) along the driveway. For the Drillfield path $\mathcal{P}, D_{\mathcal{P}}=1228$ $\mathrm{m}$. We assume the WCV travels at a speed of $V=5 \mathrm{~m} / \mathrm{s}$ along the Drillfield driveway.

The number of sensor nodes in the network will be specified later for different results. The data rate $G_{i}, i \in \mathcal{N}$ for each node is randomly generated within $[1,10] \mathrm{Kb} / \mathrm{s}$. Suppose a sensor node uses a rechargeable NiMH battery (connected to an energy receiving coil). Assume $E_{\max }=10.8 \mathrm{KJ}$ [11], and $E_{\min }=0.05 \cdot E_{\max }=540 \mathrm{~J}$. The power consumption coefficients are $\beta_{1}=50 \mathrm{~nJ} / \mathrm{b}, \beta_{2}=0.0013 \mathrm{pJ} /\left(\mathrm{b} \cdot \mathrm{m}^{4}\right)$,
TABLE II

LOCATION AND DATA RATE $G_{i}$ FOR EACH NODE IN A 25 -NODE NETWORK.

\begin{tabular}{|c|c|c||c|c|c|}
\hline $\begin{array}{c}\text { Node } \\
\text { Index }\end{array}$ & $\begin{array}{c}\text { Location } \\
(\mathrm{m})\end{array}$ & $\begin{array}{c}G_{i} \\
(\mathrm{~Kb} / \mathrm{s})\end{array}$ & $\begin{array}{c}\text { Node } \\
\text { Index }\end{array}$ & $\begin{array}{c}\text { Location } \\
(\mathrm{m})\end{array}$ & $\begin{array}{c}G_{i} \\
(\mathrm{~Kb} / \mathrm{s})\end{array}$ \\
\hline 1 & $(626.0,236.1)$ & 1 & 14 & $(247.6,181.6)$ & 7 \\
2 & $(623.3,235.6)$ & 7 & 15 & $(245.9,180.4)$ & 4 \\
3 & $(624.0,237.2)$ & 1 & 16 & $(247.7,181.0)$ & 10 \\
4 & $(625.6,237.1)$ & 1 & 17 & $(220.5,118.0)$ & 5 \\
5 & $(460.8,357.8)$ & 1 & 18 & $(220.5,121.1)$ & 2 \\
6 & $(462.6,361.9)$ & 4 & 19 & $(219.5,119.8)$ & 7 \\
7 & $(459.8,359.0)$ & 2 & 20 & $(220.7,118.3)$ & 6 \\
8 & $(461.1,359.0)$ & 3 & 21 & $(328.8,12.2)$ & 2 \\
9 & $(435.7,337.8)$ & 2 & 22 & $(335.2,13.2)$ & 9 \\
10 & $(433.3,337.7)$ & 4 & 23 & $(334.2,13.0)$ & 4 \\
11 & $(435.2,338.5)$ & 5 & 24 & $(333.2,13.9)$ & 8 \\
12 & $(434.8,337.4)$ & 5 & 25 & $(331.5,13.7)$ & 8 \\
13 & $(245.1,180.3)$ & 1 & & & \\
\hline
\end{tabular}

TABLE III

INDEX OF STOPPING POINT ALONG THE PATH, LOCATION AND TIME SPENT AT EACH STOPPING POINT FOR THE 25-NODE NETWORK.

\begin{tabular}{|c|c|c||c|c|c|}
\hline $\begin{array}{c}\text { Visit } \\
\text { Order }\end{array}$ & $\begin{array}{c}\text { Location } \\
(\mathrm{m})\end{array}$ & $\begin{array}{c}\omega(p) \\
(\mathrm{s})\end{array}$ & $\begin{array}{c}\text { Visit } \\
\text { Order }\end{array}$ & $\begin{array}{c}\text { Location } \\
(\mathrm{m})\end{array}$ & $\begin{array}{c}\omega(p) \\
(\mathrm{s})\end{array}$ \\
\hline 1 & $(625.7,235.3)$ & 23 & 6 & $(221.0,119.4)$ & 219 \\
2 & $(461.1,357.4)$ & 358 & 7 & $(329.3,11.9)$ & 2 \\
3 & $(464.5,360.2)$ & 9 & 8 & $(332.4,12.1)$ & 9 \\
4 & $(435.4,336.2)$ & 98 & 9 & $(333.9,12.4)$ & 2318 \\
5 & $(247.3,179.3)$ & 42 & & & \\
\hline
\end{tabular}

and $\rho=50 \mathrm{~nJ} / \mathrm{b}$ [6], [21]. Assume the path loss index is $\alpha=4$. For the charging efficiency function $\mu\left(D_{i B}\right)$, through curve fitting to [10, Fig. 3], we obtain $\mu\left(D_{i B}\right)=$ $-0.0958 D_{i B}^{2}-0.0377 D_{i B}+1.0$. Letting $U_{\max }=5 \mathrm{~W}$ and $\delta=1 \mathrm{~W}$, we have $D_{\delta}=2.7 \mathrm{~m}$ for a maximum distance of effective charging. For $\epsilon$, we set it to 0.05 .

\section{B. Case Study}

25-node Network. We first present results for a 25-node sensor network. The location of each node and its data rate are given in Table II. Applying our $(1-\epsilon)$-optimal algorithm, we find that the algorithm finds a solution after the third iteration. In this solution, we have $\tau=17.29 \mathrm{~h}, \tau_{\text {vac }}=16.29 \mathrm{~h}$, and the objective value is $94.21 \% .^{9}$ Since the total traveling time along path $\mathcal{P}$ is $1228 / 5=245.6 \mathrm{~s} \approx 0.07 \mathrm{~h}$, we have that the total stopping time for charging is $17.29-16.29-0.07=0.93 \mathrm{~h}$.

Upon termination, there are a total of 316 segments (corresponding to 316 logical points). However, the WCV only made 9 stops among these segments, and merely traversed all the other segments without stopping. For illustration purpose, we use a physical point $(x, y)$ within the corresponding segment to represent the segment where the WCV made a stop. These stopping points are marked with flags in Fig. 4, and the location and the amount of time at each stop are given in Table III. Note that the number of stops for the WCV is much fewer than the number of sensor nodes due to multi-node charging. For example, the WCV charges nodes $1,2,3$, and 4 at the same time when it stops at the first point $(625.7,235.3)$. Also, it is possible that a node may be charged more than once

\footnotetext{
${ }^{9}$ The objective value achieved by the upper bound solution is $95.91 \%$. This means that our solution achieves an objective value that is at least $0.9421 / 0.9591=99.0 \%$ of the optimum
} 
TABLE IV

LOCATION AND DATA RATE $G_{i}$ FOR EACH NODE IN A 50-NODE NETWORK.

\begin{tabular}{|c|c|c||c|c|c|}
\hline $\begin{array}{c}\text { Node } \\
\text { Index }\end{array}$ & $\begin{array}{c}\text { Location } \\
(\mathrm{m})\end{array}$ & $\begin{array}{c}G_{i} \\
(\mathrm{~Kb} / \mathrm{s})\end{array}$ & $\begin{array}{c}\text { Node } \\
\text { Index }\end{array}$ & $\begin{array}{c}\text { Location } \\
(\mathrm{m})\end{array}$ & $\begin{array}{c}G_{i} \\
(\mathrm{~Kb} / \mathrm{s})\end{array}$ \\
\hline 1 & $(608.8,384.8)$ & 9 & 26 & $(229.4,69.6)$ & 5 \\
2 & $(225.1,145.9)$ & 10 & 27 & $(260.9,31.7)$ & 10 \\
3 & $(229.8,158.0)$ & 5 & 28 & $(546.9,402.6)$ & 4 \\
4 & $(306.2,232.5)$ & 3 & 29 & $(649.1,322.7)$ & 2 \\
5 & $(519.4,397.8)$ & 9 & 30 & $(650.8,305.6)$ & 1 \\
6 & $(333.5,14.3)$ & 1 & 31 & $(582.4,398.5)$ & 2 \\
7 & $(234.5,60.6)$ & 2 & 32 & $(566.6,402.1)$ & 7 \\
8 & $(634.3,358.6)$ & 8 & 33 & $(650.0,303.3)$ & 7 \\
9 & $(230.9,67.3)$ & 8 & 34 & $(523.7,151.6)$ & 9 \\
10 & $(515.8,397.5)$ & 1 & 35 & $(279.4,21.2)$ & 5 \\
11 & $(575.6,400.2)$ & 8 & 36 & $(587.9,396.3)$ & 5 \\
12 & $(222.4,90.0)$ & 6 & 37 & $(456.8,354.8)$ & 4 \\
13 & $(219.5,113.4)$ & 10 & 38 & $(315.8,13.4)$ & 10 \\
14 & $(494.6,385.8)$ & 2 & 39 & $(482.3,116.8)$ & 3 \\
15 & $(296.8,15.5)$ & 4 & 40 & $(232.7,63.7)$ & 4 \\
16 & $(639.0,257.0)$ & 9 & 41 & $(434.3,76.0)$ & 1 \\
17 & $(649.6,288.1)$ & 10 & 42 & $(311.8,13.7)$ & 2 \\
18 & $(352.5,19.3)$ & 2 & 43 & $(646.4,331.7)$ & 2 \\
19 & $(322.2,11.9)$ & 2 & 44 & $(629.4,365.5)$ & 9 \\
20 & $(620.1,230.2)$ & 1 & 45 & $(614.0,381.0)$ & 8 \\
21 & $(380.7,34.0)$ & 5 & 46 & $(305.9,231.2)$ & 2 \\
22 & $(599.5,391.3)$ & 4 & 47 & $(531.0,400.6)$ & 2 \\
23 & $(307.9,12.8)$ & 4 & 48 & $(229.5,70.1)$ & 7 \\
24 & $(277.4,22.0)$ & 4 & 49 & $(235.2,59.2)$ & 8 \\
25 & $(332.6,12.8)$ & 2 & 50 & $(528.7,401.3)$ & 5 \\
\hline
\end{tabular}

TABLE V

INDEX OF STOPPING POINT ALONG THE PATH, LOCATION AND TIME SPENT AT EACH STOPPING POINT FOR THE 50-NODE NETWORK.

\begin{tabular}{|c|c|c||c|c|c|}
\hline $\begin{array}{c}\text { Visit } \\
\text { Order }\end{array}$ & $\begin{array}{c}\text { Location } \\
(\mathrm{m})\end{array}$ & $\begin{array}{c}\omega(p) \\
(\mathrm{s})\end{array}$ & $\begin{array}{c}\text { Visit } \\
\text { Order }\end{array}$ & $\begin{array}{c}\text { Location } \\
(\mathrm{m})\end{array}$ & $\begin{array}{c}\omega(p) \\
(\mathrm{s})\end{array}$ \\
\hline 1 & $(651.7,303.1)$ & 178 & 23 & $(615.0,380.1)$ & 78 \\
2 & $(628.9,366.4)$ & 236 & 24 & $(223.1,90.2)$ & 27 \\
3 & $(590.2,394.9)$ & 96 & 25 & $(353.3,17.0)$ & 22 \\
4 & $(576.7,399.5)$ & 56 & 26 & $(547.6,402.5)$ & 38 \\
5 & $(566.4,401.6)$ & 260 & 27 & $(308.3,12.3)$ & 19 \\
6 & $(515.0,395.6)$ & 7 & 28 & $(647.3,332.0)$ & 10 \\
7 & $(229.9,69.8)$ & 254 & 29 & $(649.6,322.9)$ & 9 \\
8 & $(232.1,65.1)$ & 24 & 30 & $(619.3,229.0)$ & 2675 \\
9 & $(295.8,14.5)$ & 18 & 31 & $(332.8,12.3)$ & 2041 \\
10 & $(310.5,12.1)$ & 23 & 32 & $(482.1,115.2)$ & 1591 \\
11 & $(316.5,11.5)$ & 59 & 33 & $(634.6,358.8)$ & 529 \\
12 & $(433.9,75.3)$ & 1067 & 34 & $(226.7,145.5)$ & 175 \\
13 & $(608.6,384.8)$ & 48 & 35 & $(584.5,397.1)$ & 28 \\
14 & $(277.9,21.1)$ & 406 & 36 & $(230.4,68.6)$ & 50 \\
15 & $(524.7,150.4)$ & 177 & 37 & $(234.7,60.7)$ & 49 \\
16 & $(307.8,230.5)$ & 130 & 38 & $(220.8,114.4)$ & 106 \\
17 & $(381.8,32.4)$ & 107 & 39 & $(650.6,287.4)$ & 871 \\
18 & $(260.8,31.5)$ & 76 & 40 & $(458.2,355.0)$ & 27 \\
19 & $(519.6,397.2)$ & 45 & 41 & $(600.2,390.0)$ & 20 \\
20 & $(640.3,256.3)$ & 51 & 42 & $(495.7,385.4)$ & 47 \\
21 & $(231.8,157.1)$ & 40 & 43 & $(322.3,11.6)$ & 33 \\
22 & $(531.0,400.3)$ & 55 & & & \\
\hline
\end{tabular}

in a cycle. For example, node 25 is charged when the WCV stops at both the eighth point $(332.4,12.1)$ and the ninth point $(333.9,12.4)$.

For illustration, we show flow routing (and rates) at node 12 and node 22 when the WCV stays at the second segment:

(i) At node 12, self-generated rate $R_{12}=5.0$; incoming flow rates: $f_{13,12}\left(p_{2}\right)=3.0, f_{14,12}\left(p_{2}\right)=7.0$, $f_{15,12}\left(p_{2}\right)=9.0$, and $f_{16,12}\left(p_{2}\right)=23.0$; outgoing flow rates: $f_{12,8}\left(p_{2}\right)=47$, all in $\mathrm{kb} / \mathrm{s}$.

(ii) At node 22, self-generated rate $R_{22}=9.0$; incoming flow rates: $f_{21,22}\left(p_{2}\right)=2.0$ and $f_{25,22}\left(p_{2}\right)=8.0$;
TABLE VI

THE NUMBER OF ITERATIONS FOR 20 RANDOMLY GENERATED 25-NODE NETWORKS AND 20 RANDOMLY GENERATED 50-NODE NETWORKS.

\begin{tabular}{|c|c|c||c|c|c|}
\hline \multirow{2}{*}{$\begin{array}{c}\text { Network } \\
\text { instance }\end{array}$} & \multicolumn{2}{|c|}{ Number of iterations } & \multirow{2}{*}{$\begin{array}{c}\text { Network } \\
\text { instance }\end{array}$} & \multicolumn{2}{c|}{ Number of iterations } \\
\cline { 5 - 6 } & 25 -node & 50 -node & 25-node & 50-node \\
\hline 1 & 4 & 5 & 11 & 4 & 5 \\
\hline 2 & 1 & 4 & 12 & 7 & 4 \\
\hline 3 & 3 & 3 & 13 & 4 & 6 \\
\hline 4 & 3 & 7 & 14 & 4 & 4 \\
\hline 5 & 2 & 6 & 15 & 3 & 4 \\
\hline 6 & 3 & 5 & 16 & 3 & 6 \\
\hline 7 & 5 & 4 & 17 & 5 & 5 \\
\hline 8 & 3 & 4 & 18 & 4 & 3 \\
\hline 9 & 4 & 5 & 19 & 5 & 3 \\
\hline 10 & 3 & 3 & 20 & 2 & 4 \\
\hline
\end{tabular}

outgoing flow rate: $f_{22, B}\left(p_{2}\right)=19.0$, all in $\mathrm{kb} / \mathrm{s}$.

Due to space limitation, we cannot list data flows among all sensor nodes and the mobile base station. Additional results can be found in [23].

50-node Network. We next present results for a 50-node sensor network. The location of each node and its data rate are given in Table IV. Our $(1-\epsilon)$-optimal algorithm finds a solution after the third iteration. In this solution, we have $\tau=$ $90.16 \mathrm{~h}, \tau_{\text {vac }}=86.81 \mathrm{~h}$, and the objective value is $96.28 \% .{ }^{10}$ Since the total traveling time along path $\mathcal{P}$ is approximately $0.07 \mathrm{~h}$, we have that the total stopping time for charging is $90.16-86.81-0.07=3.28 \mathrm{~h}$.

Upon termination, there are a total of 300 segments (corresponding to 300 logical points). Among these segments, the WCV made 43 stops among these segments, and merely traversed all the other segments without stopping. The location of a physical point $(x, y)$ within the corresponding segment and the amount of time at each stop are given in Table $\mathrm{V}$. Data flow routing is not included here due to space limitation.

\section{Convergence Speed}

To see the convergence speed of our algorithm, we study 20 randomly generated 25-node networks and 20 randomly generated 50-node networks, respectively. Table VI shows the number of iterations for each instance. For 25-node networks, the average number of iterations and standard deviation are 3.60 and 1.31, respectively. For 50-node networks, they are 4.50 and 1.15 , respectively.

\section{CONCLUSIONS}

This paper explored the interesting and challenging problem of using a single mobile platform (WCV) to carry both wireless charger (for WET) and base station (for data collection) in a sensor network. The goal was to minimize energy consumption of the entire system under the constraints that (i) none of the sensor nodes runs out of energy, and (ii) all data collected by the sensor nodes are relayed to the base station in real time. We developed a mathematical model for this problem and found that it is a highly complex problem involving timedependent variables. Instead of studying the original problem

\footnotetext{
${ }^{10}$ The objective value achieved by the upper bound solution is $97.10 \%$, which shows that our solution achieves an objective value that is at least $0.9628 / 0.9710=99.2 \%$ of the optimum
} 
formulation (OPT-t), we showed that it is sufficient to study a special subproblem (OPT-s) that only involves locationdependent variables. Subsequently, we developed a provably near-optimal solution to OPT-s.

A noticeable limitation of this work is that the traveling path $\mathcal{P}$ for the WCV is pre-planned. Finding an optimal path $\mathcal{P}$ will add another degree of freedom in problem space and make the problem even more difficult. Nevertheless, this is an important problem to consider and we will investigate this problem in our future research.

\section{APPENDIX}

Proof of Lemma 2: Our proof is based on construction. Given a pre-planned path $\mathcal{P}$ and $\psi_{\text {opT-t }}=\left(\tau, \tau_{\text {vac }}, \omega(p)\right.$, $\left.(x, y)(t), f_{i j}(t), f_{i B}(t), C_{i B}(t), r_{i}(t)\right)$, we let the WCV follow the same path $\mathcal{P}$ and construct a solution $\psi_{\text {OPT-s }}=\left(\hat{\tau}, \hat{\tau}_{\text {vac }}, \hat{\omega}(p)\right.$, $\left.\hat{f}_{i j}(p), \hat{f}_{i B}(p), \hat{r}_{i}(p)\right)$ by letting $\hat{\tau}=\tau, \hat{\tau}_{\text {vac }}=\tau_{\text {vac }}, \hat{\omega}(p)=$ $\omega(p)$. For the rest of the solution, we consider two cases:

- If $\omega(p)>0$, i.e., the WCV makes a stop at point $p$, we set

$$
\begin{aligned}
\hat{f}_{i j}(p) & =\frac{\int_{\{t \in[0, \tau]:(x, y)(t)=p\}} f_{i j}(t) \mathrm{d} t}{\hat{\omega}(p)} \\
\hat{f}_{i B}(p) & =\frac{\int_{\{t \in[0, \tau]:(x, y)(t)=p\}} f_{i B}(t) \mathrm{d} t}{\hat{\omega}(p)} .
\end{aligned}
$$

- If $\omega(p)=0$, i.e., the WCV merely traverses $p$ (even multiple times) without stopping, we set

$$
\begin{aligned}
\hat{f}_{i j}(p) & =\frac{\sum_{s \in \mathcal{Z}(p)} f_{i j}(t(s))}{|\mathcal{Z}(p)|} \\
\hat{f}_{i B}(p) & =\frac{\sum_{s \in \mathcal{Z}(p)} f_{i B}(t(s))}{|\mathcal{Z}(p)|}
\end{aligned}
$$

where $\mathcal{Z}(p)$ denotes the set of distances $s \in\left[0, D_{\mathcal{P}}\right]$ that correspond to the same location $p$, and $t(s)$ denoted time instance at distance $s$.

For either $\omega(p)>0$ or $\omega(p)=0$, we set $\hat{r}_{i}(p)$ by (11).

Now, all we need to do is to verify that $\psi_{\text {OPT-s }}$ is a feasible solution to Problem OPT-s and to show that the objective value of $\psi_{\text {OPT-s }}$ is the same as that of $\psi_{\text {OPT-t }}$. To show the feasibility of $\psi_{\text {OPT-s }}$, we need to verify that $\psi_{\text {OPT-s }}$ satisfies constraints (2), (10), (11), (13), and (14). Since (2) is unchanged, and $\hat{\tau}=\tau$, $\hat{\tau}_{\text {vac }}=\tau_{\text {vac }}, \hat{\omega}(p)=\omega(p)$, we have that $\psi_{\text {OPT-s }}$ satisfies (2). To verify (10) for those $p \in \mathcal{P}$ with $\omega(p)>0$, we have

$$
\begin{aligned}
& \sum_{k \in \mathcal{N}}^{k \neq i} \hat{f}_{k i}(p)+G_{i} \\
= & \sum_{k \in \mathcal{N}}^{k \neq i} \frac{\int_{\{t \in[0, \tau]:(x, y)(t)=p\}} f_{k i}(t) \mathrm{d} t}{\hat{\omega}(p)} \\
& +\frac{\int_{\{t \in[0, \tau]:(x, y)(t)=p\}} G_{i} \mathrm{~d} t}{\hat{\omega}(p)} \\
= & \frac{\int_{\{t \in[0, \tau]:(x, y)(t)=p\}}\left[\sum_{k \in \mathcal{N}}^{k \neq i} f_{k i}(t)+G_{i}\right] \mathrm{d} t}{\hat{\omega}(p)} \\
= & \frac{\int_{\{t \in[0, \tau]:(x, y)(t)=p\}}\left[\sum_{j \in \mathcal{N}}^{j \neq i} f_{i j}(t)+f_{i B}(t)\right] \mathrm{d} t}{\hat{\omega}(p)}
\end{aligned}
$$

$$
\begin{aligned}
= & \sum_{j \in \mathcal{N}}^{j \neq i} \frac{\int_{\{t \in[0, \tau]:(x, y)(t)=p\}} f_{i j}(t) \mathrm{d} t}{\hat{\omega}(p)} \\
& +\frac{\int_{\{t \in[0, \tau]:(x, y)(t)=p\}} f_{i B}(t) \mathrm{d} t}{\hat{\omega}(p)} \\
= & \sum_{j \in \mathcal{N}}^{j \neq i} \hat{f}_{i j}(p)+\hat{f}_{i B}(p),
\end{aligned}
$$

where the first equality holds by (18), the third equality holds since $\psi_{\text {OPT-t }}$ satisfies (3), and the last equality holds by (18) and (19).

Similarly, to verify (10) for those $p \in \mathcal{P}$ with $\omega(p)=0$, we have

$$
\begin{aligned}
& \sum_{k \in \mathcal{N}}^{k \neq i} \hat{f}_{k i}(p)+G_{i} \\
= & \sum_{k \in \mathcal{N}}^{k \neq i} \frac{\sum_{s \in \mathcal{Z}(p)} f_{k i}(t(s))}{|\mathcal{Z}(p)|}+\frac{\sum_{s \in \mathcal{Z}(p)} G_{i}}{|\mathcal{Z}(p)|} \\
= & \frac{\sum_{s \in \mathcal{Z}(p)}\left[\sum_{k \in \mathcal{N}}^{k \neq i} f_{k i}(t(s))+G_{i}\right]}{|\mathcal{Z}(p)|} \\
= & \frac{\sum_{s \in \mathcal{Z}(p)}\left[\sum_{j \in \mathcal{N}}^{j \neq i} f_{i j}(t(s))+f_{i B}(t(s))\right]}{|\mathcal{Z}(p)|} \\
= & \sum_{j \in \mathcal{N}}^{j \neq i} \frac{\sum_{s \in \mathcal{Z}(p)} f_{i j}(t(s))}{|\mathcal{Z}(p)|}+\frac{\sum_{s \in \mathcal{Z}(p)} f_{i B}(t(s))}{|\mathcal{Z}(p)|} \\
= & \sum_{j \in \mathcal{N}}^{j \neq i} \hat{f}_{i j}(p)+\hat{f}_{i B}(p),
\end{aligned}
$$

where the first equality holds by (20), the third equality holds since $\psi_{\text {OPT-t }}$ satisfies (3), and the last equality holds by (20) and (21).

Since $\hat{r}_{i}(p)$ is set by (11), $\psi_{\text {OPT-s }}$ must satisfy constraint (11). To verify that $\psi_{\text {OPT-s }}$ satisfies constraints (13) and (14), we first show that, for $p \in \mathcal{P}$ with $\omega(p)>0$, we have

$$
\begin{aligned}
& \hat{r}_{i}(p)=\rho \sum_{k \in \mathcal{N}}^{k \neq i} \hat{f}_{k i}(p)+\sum_{j \in \mathcal{N}}^{j \neq i} C_{i j} \cdot \hat{f}_{i j}(p)+C_{i B}(p) \cdot \hat{f}_{i B}(p) \\
& =\rho \sum_{k \in \mathcal{N}}^{k \neq i} \frac{\int_{\{t \in[0, \tau]:(x, y)(t)=p\}} f_{k i}(t) \mathrm{d} t}{\hat{\omega}(p)} \\
& +\sum_{j \in \mathcal{N}}^{j \neq i} C_{i j} \cdot \frac{\int_{\{t \in[0, \tau]:(x, y)(t)=p\}} f_{i j}(t) \mathrm{d} t}{\hat{\omega}(p)} \\
& +C_{i B}(p) \cdot \frac{\int_{\{t \in[0, \tau]:(x, y)(t)=p\}} f_{i B}(t) \mathrm{d} t}{\hat{\omega}(p)} \\
& =\frac{\int_{\{t \in[0, \tau]:(x, y)(t)=p\}}\left[\rho \cdot \sum_{k \in \mathcal{N}}^{k \neq i} f_{k i}(t)\right.}{\hat{\omega}(p)} \\
& \frac{\left.+\sum_{j \in \mathcal{N}}^{j \neq i} C_{i j} \cdot f_{i j}(t)+C_{i B}(p) \cdot f_{i B}(t)\right] \mathrm{d} t}{\hat{\omega}(p)} \\
& =\frac{\int_{\{t \in[0, \tau]:(x, y)(t)=p\}} r_{i}(t) \mathrm{d} t}{\hat{\omega}(p)},
\end{aligned}
$$

where the first equality holds since $\hat{r}_{i}(p)$ is set by (11), the second equality holds by (18) and (19), and the last equality holds since $\psi_{\text {OPT-t }}$ satisfies (5). Similarly, for $p \in \mathcal{P}$ with $\omega(p)=$ 
0 , we have

$$
\hat{r}_{i}(p)=\frac{\sum_{s \in \mathcal{Z}(p)} r_{i}(t(s))}{|\mathcal{Z}(p)|} .
$$

By (22) and (23), we can verify (13) as follows:

$$
\begin{aligned}
& \hat{r}_{i}\left(p_{\text {vac }}\right) \cdot \hat{\tau}_{\text {vac }}+\sum_{p \in \mathcal{P}, p \neq p_{\text {vac }}}^{\hat{\omega}(p)>0} \hat{r}_{i}(p) \cdot \hat{\omega}(p) \\
& +\int_{s \in\left[0, D_{\mathrm{P}}\right]}^{\hat{\omega}(p(s))=0} W \cdot \hat{r}_{i}(p(s)) \mathrm{d} s \\
& =\frac{\int_{\left\{t \in[0, \tau]:(x, y)(t)=p_{\mathrm{vac}\}}\right.} r_{i}(t) \mathrm{d} t}{\hat{\tau}_{\text {vac }}} \cdot \hat{\tau}_{\text {vac }} \\
& +\sum_{p \in \mathcal{P}, p \neq p_{\text {vac }}}^{\hat{\omega}(p)>0} \frac{\int_{\{t \in[0, \tau]:(x, y)(t)=p\}} r_{i}(t) \mathrm{d} t}{\hat{\omega}(p)} \cdot \hat{\omega}(p) \\
& +\int_{s \in\left[0, D_{\mathrm{P}}\right]}^{\hat{\tau}_{\mathrm{B}}(p(s))=0} W \cdot \frac{\sum_{s \in \mathcal{Z}(p)} r_{i}(t(s))}{|\mathcal{Z}(p)|} \mathrm{d} s \\
& =\int_{\left\{t \in[0, \tau]:(x, y)(t)=p_{\text {vac }}\right\}} r_{i}(t) \mathrm{d} t \\
& +\sum_{p \in \mathcal{P}, p \neq p_{\text {vac }}}^{\omega(p)>0} \int_{\{t \in[0, \tau]:(x, y)(t)=p\}} r_{i}(t) \mathrm{d} t \\
& +\int_{p \in \mathcal{P}}^{\omega(p)=0} \sum_{s \in \mathcal{Z}(p)} W \cdot \frac{\sum_{s \in \mathcal{Z}(p)} r_{i}(t(s))}{|\mathcal{Z}(p)|} \mathrm{d} s \\
& =\int_{\left\{t \in[0, \tau]:(x, y)(t)=p_{\text {vac }}\right\}} r_{i}(t) \mathrm{d} t \\
& +\sum_{p \in \mathcal{P}, p \neq p_{\text {vac }}}^{\omega(p)>0} \int_{\{t \in[0, \tau]:(x, y)(t)=p\}} r_{i}(t) \mathrm{d} t \\
& +\int_{p \in \mathcal{P}}^{\omega(p)=0} \int_{\{t \in[0, \tau]:(x, y)(t)=p\}} r_{i}(t) \mathrm{d} t \\
& =\int_{0}^{\tau} r_{i}(t) \mathrm{d} t \leq \sum_{p \in \mathcal{P}}^{\omega(p)>0} U_{i B}(p) \cdot \omega(p) \\
& =\sum_{p \in \mathcal{P}}^{\hat{\omega}(p)>0} U_{i B}(p) \cdot \hat{\omega}(p),
\end{aligned}
$$

where the first equality holds by (22) and (23), the second equality holds due to $\omega(p)=\hat{\omega}(p)$, the third equality holds due to the following equation:

$$
\begin{aligned}
& \sum_{s \in \mathcal{Z}(p)} W \cdot \frac{\sum_{s \in \mathcal{Z}(p)} r_{i}(t(s))}{|\mathcal{Z}(p)|} \mathrm{d} s \\
= & |\mathcal{Z}(p)| \cdot \frac{d t}{d s} \cdot \frac{\sum_{s \in \mathcal{Z}(p)} r_{i}(t(s))}{|\mathcal{Z}(p)|} \mathrm{d} s \\
= & \sum_{s \in \mathcal{Z}_{(p)}} r_{i}(t(s)) \mathrm{d} t \\
= & \int_{\{t \in[0, \tau]:(x, y)(t)=p\}} r_{i}(t) \mathrm{d} t,
\end{aligned}
$$

the fourth equality holds since every possible location along $\mathcal{P}$ is covered, the fifth inequality holds since $\psi_{\text {OPT-t }}$ satisfies (8), and the last equality holds due to $\hat{\omega}(p)=\omega(p)$. The proof for (14) is very similar to the above proof for (13) and thus is omitted to conserve space.
We have thus shown that $\psi_{\text {OPT-s }}$ is a feasible solution to problem OPT-s. We also have

$$
\frac{\hat{\tau}_{\text {vac }}}{\hat{\tau}}=\frac{\tau_{\text {vac }}}{\tau},
$$

where the equality holds simply due to $\hat{\tau}=\tau$ and $\hat{\tau}_{\text {vac }}=\tau_{\text {vac }}$. That is, the objective value of $\psi_{\text {OPT-s }}$ is equal to that of $\psi_{\text {OPT-t }}$. This completes our proof.

\section{REFERENCES}

[1] S. Basagni, A. Carosi, E. Melachrinoudis, C. Petrioli, and Z.M. Wang, "A new MILP formulation and distributed protocols for wireless sensor networks lifetime maximization," in Proc. IEEE ICC, Istanbul, Turkey, June 2006, pp. 3517-3524.

[2] J. Chang and L. Tassiulas, "Maximum lifetime routing in wireless sensor networks," IEEE/ACM Trans. on Networking, vol. 12, no. 4, pp. 609619, Aug. 2004.

[3] R. Doost, K.R. Chowdhury, and M.D. Felice, "Routing and link layer protocol design for sensor networks with wireless energy transfer," in Proc. IEEE GLOBECOM, Miami, FL, Dec. 2010, pp. 1-5.

[4] M. Erol-Kantarci, and H.T. Mouftah, "Suresense: Sustainable wireless rechargeable sensor networks for the smart grid," IEEE Wireless Commun., vol. 19, no. 3, pp. 30-36, June. 2012.

[5] S. He, J. Chen, F. Jiang, D.K.Y. Yau, G. Xing, and Y. Sun, "Energy provisioning in wireless rechargeable sensor networks," in Proc. IEEE INFOCOM, Shanghai, China, Apr. 2011, pp. 2006-2014.

[6] W.B. Heinzelman, "Application-specific protocol architectures for wireless networks," Ph.D. Dissertation, Dept. Elect. Eng. Comput. Sci., MIT, Cambridge, MA, Jun. 2000.

[7] Y.T. Hou, Y. Shi, and H.D. Sherali, "Rate allocation and network lifetime problems for wireless sensor networks," IEEE/ACM Trans. on Networking, vol. 16, no. 2, pp. 321-334, Apr. 2008.

[8] S. Jain, K. Fall, and R. Patra, "Routing in a delay tolerant network," in Proc. ACM SIGCOMM, pp. 145-158, Portland, OR, Aug. 30-Sep. 3, 2004.

[9] A. Kurs, A. Karalis, R. Moffatt, J.D. Joannopoulos, P. Fisher, and M. Soljacic, "Wireless power transfer via strongly coupled magnetic resonances," Science, vol. 317, no. 5834, pp. 83-86, July 2007.

[10] A. Kurs, R. Moffatt, and M. Soljacic, "Simultaneous mid-range power transfer to multiple devices," Appl. Phys. Lett., vol. 96, pp. 044102-1044102-3, Jan. 2010.

[11] D. Linden and T.B. Reddy (eds.), Handbook of Batteries, 3rd ed., Chapter 1, McGraw-Hill, 2002.

[12] J. Luo and J.-P. Huabux, "Joint mobility and routing for lifetime elongation in wireless sensor networks," in Proc. IEEE INFOCOM, Miami, FL, March 13-17, 2005, pp. 1735-1746.

[13] J. Luo and J.-P. Huabux, "Joint sink mobility and routing to maximize the lifetime of wireless sensor networks: The case of constrained mobility," IEEE/ACM Trans. on Networking, vol. 18, no. 3, pp. 871884, June 2010.

[14] PowerCast, http://www.powercastco.com.

[15] A. Sample, D. Yaniel, P. Powledge, A. Mamishev, and J. Smith, "Design of an RFID-based battery-free programmable sensing platform," IEEE Trans. on Instrumentation and Measurement, vol. 57, no. 11, pp. 26082615, Nov. 2008

[16] R. C. Shah, S. Roy, S. Jain, and W. Brunette, "Data MULEs: Modeling a three-tier architecture for sparse sensor networks," in Proc. of First IEEE International Workshop on Sensor Network Protocols and Applications (SNPA), pp. 30-41, Anchorage, AK, May 2003.

[17] Y. Shi and Y.T. Hou, "Some fundamental results on base station movement problem for wireless sensor networks," IEEE/ACM Trans. on Networking, vol. 20, issue 4, pp. 1054-1067, Aug. 2012.

[18] B. Tong, Z. Li, G. Wang, and W. Zhang, "How wireless power charging technology affects sensor network deployment and routing," in Proc. IEEE ICDCS, Genoa, Italy, Jun. 2010, pp. 438-447.

[19] U.S. Environmental Protection Agency and U.S. Department of Energy, "2013 Scion iQ EV," URL: http://www.fueleconomy.gov.

[20] W. Wang, V. Srinivasan, and K.C. Chua, "Extending the lifetime of wireless sensor networks through mobile relays," IEEE/ACM Trans. on Networking, vol. 16, no. 5, pp. 1108-1120, Oct. 2008.

[21] L. Xie, Y. Shi, Y.T. Hou, and H.D. Sherali, "Making sensor networks immortal: An energy-renewal approach with wireless power transfer," IEEE/ACM Trans. on Networking, vol. 20, no. 6, pp. 1748-1761, Dec. 2012. 
[22] L. Xie, Y. Shi, Y.T. Hou, W. Lou, H.D. Sherali, and S.F. Midkiff, "Multi-node wireless energy charging in sensor networks," to appear in IEEE/ACM Trans. on Networking, Digital Object Identifier; 10.1109/TNET.2014.2303979.

[23] L. Xie, Y. Shi, Y.T. Hou, W. Lou, H.D. Sherali, H. Zhou, and S.F. Midkiff, "Bundling mobile base station and wireless energy transfer: The Pre-planned Path Case," Technical Report, the Bradley Department of Electrical and Computer Engineering, Virginia Tech, Blacksburg, VA, July 2012. Available at https://sites.google.com/a/vt.edu/xie.

[24] L. Xie, Y. Shi, Y.T. Hou, and W. Lou, "Wireless power transfer and applications to sensor networks," IEEE Wireless Communications Magazine, vol. 20, no. 4, pp. 140-145, Aug. 2013. "Bundling mobile base station and wireless energy transfer: Modeling and optimization," in Proc. IEEE INFOCOM, Turin, Italy, Apr. 2013, pp. $1684-1692$.

[26] G. Xing, T. Wang, W. Jia, and M. Li, "Rendezvous design algorithms for wireless sensor networks with a mobile base station," in Proc. ACM MobiHoc, Hong Kong, China, May 27-30, 2008, pp. 231-240.

[27] M, Younis, M. Bangad and K. Akkaya, "Base-station repositioning for optimized performance of sensor networks," in Proc. IEEE Vehicular Technology Conference, Orlando, FL, Oct. 4-9, 2003, pp. 2956-2960.

[28] G.J. Zalmai, "Optimality conditions and Lagrangian duality in continuous-time nonlinear programming," Journal of Mathematical Analysis and Applications, vol. 109, no. 2, Aug. 1985, pp. 426-452.

[29] W. Zhao, M. Ammar, and E. Zegura, "A message ferrying approach for data delivery in sparse mobile ad hoc networks," in Proc. ACM MobiHoc, pp. 187-198, Tokyo, Japan, May 2004.

[30] M. Zhao, J. Li and Y. Yang, "Joint mobile energy replenishment and data gathering in wireless rechargeable sensor networks," in Proc. Inpp. $238-245$.

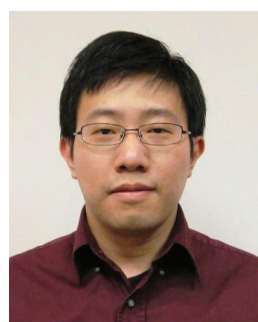

Liguang Xie (S'08-M'14) received his Ph.D. degree in Computer Engineering from Virginia Tech, Blacksburg, VA in 2013. He is currently a Senior Engineer at MicroStrategy Inc, Mclean, VA and an Adjunct Instructor in the Bradley Department of Electrical and Computer Engineering, Virginia Tech, Blacksburg, VA. His research interests include optimization and algorithm design for wireless networks, with a current focus on wireless power transfer for energy-constrained wireless networks.
[25] L. Xie, Y. Shi, Y.T. Hou, W. Lou, H.D. Sherali, and S. F. Midkiff, ternational Teletraffic Congress, San Francisco, CA, Sep. 6-8, 2011,

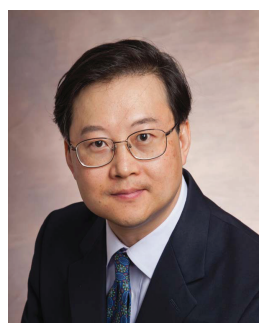

Y. Thomas Hou (S'91-M'98-SM'04-F'14) is Bradley Distinguished Professor of Electrical and Computer Engineering at Virginia Tech, Blacksburg, VA. He received his Ph.D. degree in Electrical Engineering from Polytechnic School of Engineering of New York University (NYU) in 1998. Prof. Hou's research focuses on developing innovative solutions to complex problems that arise in wireless networks. $\mathrm{He}$ is the Chair of IEEE INFOCOM Steering Committee.

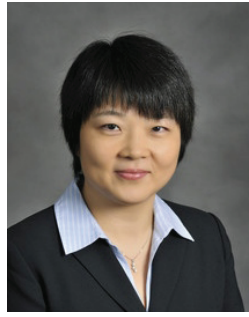

Wenjing Lou (S'01-M'03-SM'08-F'15) is a Professor in the Department of Computer Science at Virginia Tech, USA. She received her Ph.D. degree in Electrical and Computer Engineering from the University of Florida in 2003. Prof. Lou's research interests include cyber security and wireless networks. She is on the editorial boards of a number of IEEE transactions. She is the Steering Committee Chair of IEEE Conference on Communications and Network Security (CNS). Prof. Lou is currently on National Science Foundation. IPA assignment as a program director at the US

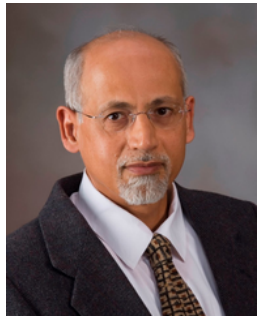

Hanif D. Sherali is a University Distinguished Professor Emeritus in the Industrial and Systems Engineering Department at Virginia Polytechnic Institute and State University. His areas of research interest are in mathematical optimization modeling, analysis, and design of algorithms for specially structured linear, nonlinear, and continuous and discrete nonconvex programs. He is a Fellow of INFORMS and IIE, and an elected member of the US National Academy of Engineering (NAE)

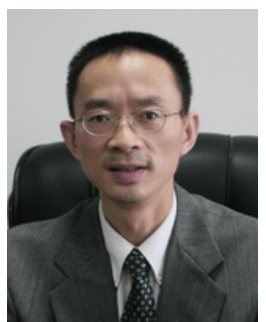

Huaibei Zhou received his B.S and M.S degrees in Physics from Wuhan University, China in 1984 and 1987, respectively, and his Ph.D. degree in Physics from the University of Maryland, College Park, in 1994. His past experience included Postdoctoral Fellow at National Institute of Standard and Technology (NIST) in Maryland (1994-1996), Senior Engineer at GE (1996-1999) and Senior Technical Manager at NEXTEL (1999-2002). He is currently a full professor in the International School of Software Engineering, Wuhan University, China. His current research interests include wireless communications and networks.

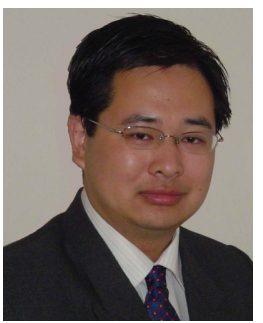

Yi Shi (S'02-M'08-SM'13) received his Ph.D. degree in Computer Engineering from Virginia Tech, Blacksburg, VA in 2007. He is currently a Senior Research Scientist at Intelligent Automation Inc., Rockville, MD, and an Adjunct Assistant Professor at Virginia Tech. His research focuses on optimization and algorithm design for wireless networks. $\mathrm{He}$ was a recipient of IEEE INFOCOM 2008 Best Paper Award and the only Best Paper Award Runner-Up of IEEE INFOCOM 2011

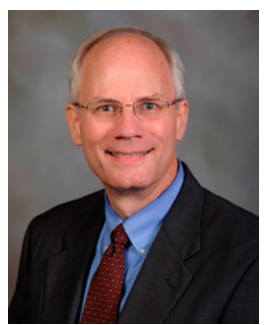

Scott F. Midkiff (S'82-M'85-SM'92) is a professor in the Bradley Department of Electrical and Computer Engineering and currently serves as Vice President for Information Technology and Chief Information Officer at Virginia Polytechnic Institute and State University (Virginia Tech), Blacksburg, VA, USA. He received his Ph.D. degree in electrical engineering from Duke University, Durham, NC. During 2006-2009, he served as a program director at the US National Science Foundation. His research interests include wireless networks, network services for pervasive computing, and cyber-physical systems. 